\title{
Gelfand models for diagram algebras
}

\author{
Tom Halverson • Mike Reeks
}

Received: 25 February 2013 / Accepted: 23 May 2014 / Published online: 24 June 2014

(C) Springer Science+Business Media New York 2014

\begin{abstract}
A Gelfand model for a semisimple algebra A over an algebraically closed field $\mathbb{K}$ is a linear representation that contains each irreducible representation of $A$ with multiplicity exactly one. We give a method of constructing these models that works uniformly for a large class of semisimple, combinatorial diagram algebras including the partition, Brauer, rook monoid, rook-Brauer, Temperley-Lieb, Motzkin, and planar rook monoid algebras. In each case, the model representation is given by diagrams acting via "signed conjugation" on the linear span of their horizontally symmetric diagrams. This representation is a generalization of the Saxl model for the symmetric group. Our method is to use the Jones basic construction to lift the Saxl model from the symmetric group to each diagram algebra. In the case of the planar diagram algebras, our construction exactly produces the irreducible representations of the algebra.
\end{abstract}

Keywords Gelfand model - Multiplicity-free representation - Symmetric group · Partition algebra $\cdot$ Brauer algebra $\cdot$ Temperley-Lieb algebra $\cdot$ Motzkin algebra . Rook monoid

\section{Introduction}

A famous consequence of Robinson-Schensted-Knuth (RSK) insertion is that the set of standard Young tableaux with $k$ boxes is in bijection with the set of involutions in the symmetric group $\mathrm{S}_{k}$ (the permutations $\sigma \in \mathrm{S}_{k}$ with $\sigma^{2}=1$ ). Since the standard

\footnotetext{
T. Halverson $(\varangle)$

Department of Mathematics, Macalester College, St. Paul, MN 55105, USA

e-mail: halverson@macalester.edu

M. Reeks

Department of Mathematics, University of Virginia, Charlottesville, VA 22904, USA

e-mail: mar3nf@virginia.edu
} 
Young tableaux index the bases for the irreducible $S_{k}$ modules, it follows that the sum of the dimensions of the irreducible $S_{k}$ modules equals the number of involutions in $\mathrm{S}_{k}$. This suggests the possibility of a representation of the symmetric group on the linear span of its involutions which decomposes into irreducible $S_{k}$ modules each with multiplicity 1. Saxl [34] and Kljačko [22] have constructed such a module under which the symmetric group acts on its involutions by a twisted, or signed, conjugation (3.5). A combinatorial construction of an analogous module was studied recently by Adin et al. [1] and extended to the rook monoid and related semigroups in [23]. A representation for which each irreducible appears with multiplicity one is called a Gelfand model (or, simply, a model), because of the work in [3] on models for complex Lie groups.

In [16], the RSK algorithm is extended to work for a large class of well known, combinatorial diagram algebras which are subalgebras of the the partition algebra. A consequence [16, (5.5)] of this algorithm is that the sum of the degrees of the irreducible representations of each of these algebras equals the number of horizontally symmetric basis diagrams in the algebra. This suggests the existence of a model representation of each of these algebras on the span of its symmetric diagrams, and the main result of this paper is to produce such a model.

Let $\mathbb{K}$ be an algebraically closed field, and let $A_{k}$ denote one of the following unital, associative $\mathbb{K}$-algebras: the partition, Brauer, rook monoid, rook-Brauer, TemperleyLieb, Motzkin, or planar rook monoid algebra. Then $A_{k}$ has a basis of diagrams and a multiplication given by diagram concatenation. The algebra $A_{k}$ depends on a parameter $x \in \mathbb{K}$ and is semisimple when $\operatorname{char}(\mathbb{K})=0$ or under special conditions on $\operatorname{char}(\mathbb{K})>0$ and for all, but a finite number of choices of $x \in \mathbb{K}$. When $A_{k}$ is semisimple, its irreducible modules are indexed by a set $\Lambda_{\mathrm{A}_{k}}$, and for $\lambda \in \Lambda_{\mathrm{A}_{k}}$, we let $A_{k}^{\lambda}$ denote the irreducible $A_{k}$-module labeled by $\lambda$. We construct, in a uniform way, an $\mathrm{A}_{k}$-module $\mathrm{M}_{\mathrm{A}_{k}}$ which decomposes as $\mathrm{M}_{\mathrm{A}_{k}} \cong \bigoplus_{\lambda \in \Lambda_{\mathrm{A}_{k}}} \mathrm{~A}_{k}^{\lambda}$.

Our model representation is constructed as follows. For a basis diagram $d$, let $d^{T}$ be its reflection across its horizontal axis and say that a diagram $t$ is symmetric if $t^{T}=t$. A basis diagram $d$ acts on a symmetric diagram $t$ by "signed conjugation": $d \cdot t=S(d, t) d t d^{T}$, where $S(d, t)$ is the sign on the permutation of the fixed blocks of $t$ induced by conjugation by $d$ (see Example 4.2 for details). In each example, our basis diagrams are assigned a rank, which is the number of blocks in the diagram that propagate from the top row to the bottom row. We let $\mathrm{M}_{\mathrm{A}_{k}}^{r}$ be the linear span of the symmetric diagrams of rank $r$ and our model is the direct sum $\mathrm{M}_{\mathrm{A}_{k}}=\oplus_{r=0}^{k} \mathrm{M}_{\mathrm{A}_{k}}^{r}$.

The diagram algebras in this paper naturally form a tower $A_{0} \subseteq A_{1} \subseteq \cdots \subseteq A_{k}$. Each algebra contains a Jones basic construction ideal $J_{k-1} \subseteq A_{k}$ such that $A_{k} \cong$ $\mathrm{J}_{k-1} \oplus \mathrm{C}_{k}$, where $\mathrm{C}_{k} \cong \mathbb{K} \mathrm{S}_{k}$ for nonplanar diagram algebras and $\mathrm{C}_{k} \cong \mathbb{K} \mathbf{1}_{k}$ for planar diagram algebras. The ideal $J_{k-1}$ is in Schur-Weyl duality with one of $A_{k-1}$ or $\mathrm{A}_{k-2}$ (depending on the specific diagram algebra), and we are able to take models for each $\mathrm{C}_{r}, 0 \leq r \leq k$, and lift them to a model for $\mathrm{A}_{k}$.

For the planar diagram algebras - the Temperley-Lieb, Motzkin, and planar rook monoid algebras - the algebra $\mathrm{C} \cong \mathbb{K} \mathbf{1}_{k}$ is trivial and the model is trivial. It follows that $\mathrm{M}_{\mathrm{A}_{k}}^{r}$ is irreducible and that signed conjugation produces a complete set of irreducible modules for the planar algebras. For the nonplanar diagram algebras, the algebra is $\mathrm{C} \cong \mathbb{K} \mathrm{S}_{k}$, and we use the Saxl model for $\mathrm{S}_{k}$. In this case $\mathrm{M}_{\mathrm{A}_{k}}^{r}$ is further graded as 
$\mathrm{M}_{\mathrm{A}_{k}}^{r}=\oplus_{f} \mathrm{M}_{\mathrm{A}_{k}}^{r, f}$, where $\mathrm{M}_{\mathrm{A}_{k}}^{r, f}$ is the linear span of symmetric diagrams of rank $r$ having $f$ "fixed blocks," and $\mathrm{M}_{\mathrm{A}_{k}}^{r, f}$ decomposes into irreducibles labeled by partitions having $f$ odd parts.

Besides being natural constructions, these representations are useful in several ways: (1) In a model representation, isotypic components are irreducible components, so projection operators map directly onto a irreducible modules without being mixed up among multiple isomorphic copies of the same module. (2) A key feature of our model is that we give the explicit action of each basis element of $\mathrm{A}_{k}$ on the basis of $\mathrm{M}_{\mathrm{A}_{k}}^{r, f}$. For small values of $k$, and for all values of $k$ in the planar case, these representations are irreducible or have few irreducible components. Thus, in practice, the model provides a natural and easy way to compute the explicit action of basis diagrams on irreducible representations. Indeed, it is through this construction that the irreducible modules for the Motzkin [2], the rook-Brauer [6], and the planar rook monoid [9] were discovered. (3) Gelfand models are useful in the study of Markov chains on related combinatorial objects; see, for example, Chapter $3 \mathrm{~F}$ of [7] and the references therein, as well as $[8,32]$.

Finally, the enumeration of symmetric diagrams in these algebras according to rank and number of fixed blocks gives rise to well known, interesting integer sequences. These combinatorics are analyzed in Sect. 5, where we work out the details of the model representation for each algebra.

\section{The partition algebra and its diagram subalgebras}

In this section, we describe the partition algebra $\mathrm{P}_{k}(x)$ over $\mathbb{K}$ with a parameter $x \in \mathbb{K}$ and realize the other diagram algebras of interest in this paper as subalgebras of $\mathrm{P}_{k}(x)$. The main results of this paper require that $\mathbb{K}$ be chosen so that $\mathrm{P}_{k}(n)$ is semisimple. For example, one may choose $\mathbb{K}$ such that $\operatorname{char}(\mathbb{K})=0$.

\subsection{The partition monoid $\mathcal{P}_{k}$}

For $k \in \mathbb{Z}_{>0}$, let $\mathcal{P}_{k}$ denote the set of set partitions of $\left\{1,2, \ldots, k, 1^{\prime}, 2^{\prime}, \ldots, k^{\prime}\right\}$. We represent a set partition $d \in \mathcal{P}_{k}$ by a diagram with vertices in the top row labeled $1, \ldots, k$ and vertices in the bottom row labeled $1^{\prime}, \ldots, k^{\prime}$. Assign edges in this diagram so that the connected components equal the underlying set partition $d$. For example, the following is a diagram $d \in \mathcal{P}_{12}$,

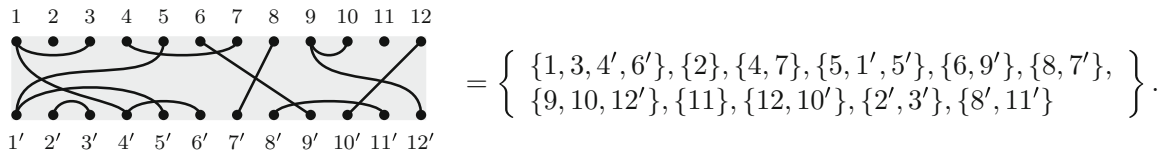

We refer to the parts of a set partition as blocks, so that the above diagram has 11 blocks. The diagram of $d$ is not unique, since it only depends on the underlying connected components. We make the following convention: if a block contains vertices from both the top row and bottom row, then we always connect the leftmost vertex in the top row 
of a block with the leftmost vertex in the bottom row of the block by a single vertical edge.

We multiply two set partition diagrams $d_{1}, d_{2} \in \mathcal{P}_{k}$ as follows. Place $d_{1}$ above $d_{2}$ and identify each vertex $j^{\prime}$ in the bottom row of $d_{1}$ with the corresponding vertex $j$ in the top row of $d_{2}$. Remove any connected components that live entirely in the middle row and let $d_{1} \circ d_{2} \in \mathcal{P}_{k}$ be the resulting diagram. For example, if

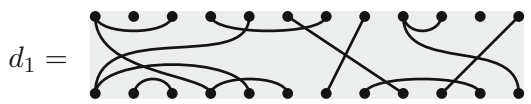

and

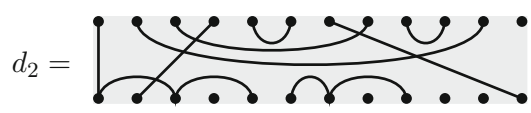

then

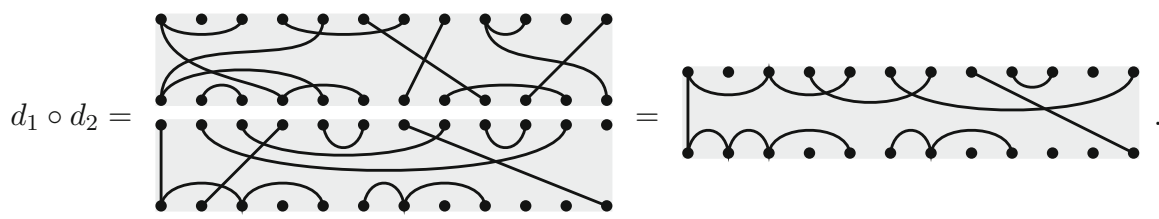

Diagram multiplication is associative and makes $\mathcal{P}_{k}$ a monoid with identity $1_{k}=\lfloor!\lfloor\ldots ! !$

\subsection{The partition algebra $\mathrm{P}_{k}(x)$}

Now let $x \in \mathbb{K}$, define $\mathrm{P}_{0}(x)=\mathbb{K}$, and for $k \geq 1$, let $\mathrm{P}_{k}(x)$ be the $\mathbb{K}$-vector space with basis $\mathcal{P}_{k}$. If $d_{1}, d_{2} \in \mathcal{P}_{k}$, let $\kappa\left(d_{1}, d_{2}\right)$ denote the number of connected components that are removed from the middle row in computing $d_{1} \circ d_{2}$, and define

$$
d_{1} d_{2}=x^{\kappa\left(d_{1}, d_{2}\right)} d_{1} \circ d_{2}
$$

In the multiplication example of the previous section $\kappa\left(d_{1}, d_{2}\right)=1$ and $d_{1} d_{2}=$ $x\left(d_{1} \circ d_{2}\right)$. This product makes $\mathrm{P}_{k}(x)$ an associative algebra with identity $\mathbf{1}_{k}$.

We say that a block $B$ in a set partition diagram $d \in \mathcal{P}_{k}$ is a propagating block if $B$ contains vertices from both the top and bottom rows of $d$; that is, both $B \cap\{1,2, \ldots, k\}$ and $B \cap\left\{1^{\prime}, 2^{\prime}, \ldots, k^{\prime}\right\}$ are nonempty. The rank of $d \in \mathcal{P}_{k}$ (also called the propagating number) is

$$
\operatorname{rank}(d)=(\text { the number of propagating blocks in } d) .
$$

The rank satisfies

$$
\operatorname{rank}\left(d_{1} d_{2}\right) \leq \min \left(\operatorname{rank}\left(d_{1}\right), \operatorname{rank}\left(d_{2}\right)\right) .
$$

For $0 \leq r \leq k$, we let $\mathrm{J}_{r} \subseteq \mathrm{P}_{k}(x)$ be the $\mathbb{K}$-span of the diagrams of rank less than or equal to $r$. Then $\mathrm{J}_{r}$ is a two-sided ideal in $\mathrm{P}_{k}(x)$, and we have a tower of ideals: $\mathrm{J}_{0} \subseteq \mathrm{J}_{1} \subseteq \mathrm{J}_{2} \subseteq \cdots \subseteq \mathrm{J}_{k}=\mathrm{P}_{k}(x)$.

The partition algebra was first defined independently by Martin [24] and Jones [21] as a higher-dimension generalization of the Temperley-Lieb algebra in statistical mechanics (see also [17] for a survey of many results on the partition algebra). 


\subsection{Subalgebras}

For each $k \in \mathbb{Z}_{>0}$, the following are subalgebras of the partition algebra $\mathrm{P}_{k}(x)$ :

$$
\mathbb{K} \mathrm{S}_{k}=\mathbb{K}-\operatorname{span}\left\{d \in \mathcal{P}_{k} \mid \operatorname{rank}(d)=k\right\},
$$

$\mathrm{B}_{k}(x)=\mathbb{K}-\operatorname{span}\left\{d \in \mathcal{P}_{k} \mid\right.$ all blocks of $d$ have size 2$\}$,

$$
\mathrm{R}_{k}=\mathbb{K} \text {-span }\left\{\begin{array}{l|l}
d \in \mathcal{P}_{k} & \begin{array}{l}
\text { all blocks of } d \text { have at most one vertex in }\{1, \ldots k\} \\
\text { and at most one vertex in }\left\{1^{\prime}, \ldots k^{\prime}\right\}
\end{array}
\end{array}\right\},
$$

$\operatorname{RB}_{k}(x)=\mathbb{K}-\operatorname{span}\left\{d \in \mathcal{P}_{k} \mid\right.$ all blocks of $d$ have size 1 or 2$\}$.

Here, $\mathbb{K S}_{k}$ is the group algebra of the symmetric group, $\mathrm{B}_{k}(x)$ is the Brauer algebra [4], $\mathrm{R}_{k}$ is the rook monoid algebra [35], and $\mathrm{RB}_{k}(x)$ is the rook-Brauer algebra $[6,26]$.

A set partition is planar if it can be represented as a diagram without edge crossings inside of the rectangle formed by its vertices. The planar partition algebra [21] is

$$
\operatorname{PP}_{k}(x)=\mathbb{K}-\operatorname{span}\left\{d \in \mathcal{P}_{k} \mid d \text { is planar }\right\}
$$

The following are the planar subalgebras of $\mathrm{P}_{k}(x)$ :

$$
\begin{aligned}
\mathbb{K}\left\{\mathbf{1}_{k}\right\} & =\mathbb{K S}_{k} \cap \mathrm{PP}_{k}(x), \quad \mathrm{TL}_{k}(x)=\mathrm{B}_{k}(x) \cap \mathrm{PP}_{k}(x), \\
\mathrm{PR}_{k} & =\mathrm{R}_{k} \cap \mathrm{PP}_{k}(x), \quad \mathrm{M}_{k}(x)=\mathrm{RB}_{k}(x) \cap \mathrm{PP}_{k}(x) .
\end{aligned}
$$

Here, $\mathrm{TL}_{k}(x)$ is the Temperley-Lieb algebra [36], $\mathrm{PR}_{k}$ is the planar rook monoid algebra [9], and $\mathrm{M}_{k}(x)$ is the Motzkin algebra [2]. The parameter $x$ does not arise when multiplying symmetric group diagrams (as there are never middle blocks to be removed). The parameter is set to be $x=1$ for the rook monoid algebra and the planar rook monoid algebra. Here are examples from each of these subalgebras:
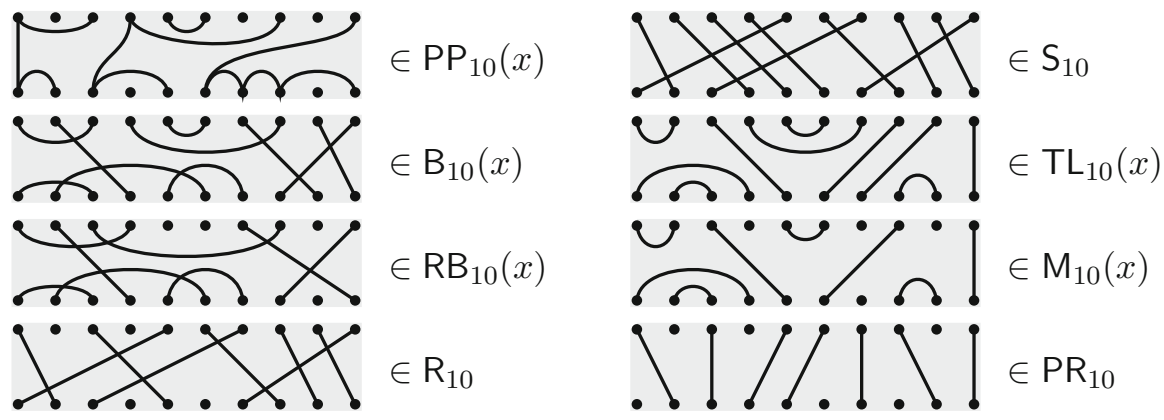

\section{A model representation of the symmetric group}

\subsection{Saxl's model characters of $\mathrm{S}_{k}$}

An involution $t \in \mathrm{S}_{k}$ is a permutation such that $t^{2}=1$. In disjoint cycle notation, involutions consist of 2-cycles and fixed points. Let $I_{k}$ be the set of involutions in $S_{k}$ 
and let $\mathbf{I}_{k}^{f}$ be the involutions in $\mathrm{S}_{k}$ which fix precisely $f$ points; that is,

$$
\mathrm{I}_{\mathrm{S}_{k}}=\left\{t \in \mathrm{S}_{n} \mid t^{2}=1\right\} \quad \text { and } \quad \mathrm{I}_{\mathrm{S}_{k}}^{f}=\left\{t \in \mathrm{S}_{n} \mid t^{2}=1 \text { and } t \text { has } f \text { fixed points }\right\} \text {. }
$$

For a fixed involution $t \in \mathrm{I}_{k}^{f}$, let $\mathrm{C}(t) \subseteq \mathrm{S}_{n}$ be the centralizer of $t$ in $\mathrm{S}_{k}$. Let $\mathrm{S}_{k}$ act on itself by conjugation so that $w \cdot \sigma=w \sigma w^{-1}$ for all $w, \sigma \in \mathrm{S}_{k}$. Then $\mathrm{C}(t)$ and $\mathrm{I}_{\mathrm{S}_{k}}^{f}$ are the stabilizer and orbit of $t$, respectively, so $\left|\mathrm{S}_{k}\right|=|\mathbf{C}(t)| \cdot\left|\mathrm{I}_{\mathrm{S}_{k}}^{f}\right|$.

If $w \in \mathrm{C}(t)$, then $w t w^{-1}=t$, so $w$ fixes $t$ but possibly permutes the fixed points of $t$. Let $\pi_{f}$ be the linear character of $\mathrm{C}(t)$ such that $\pi_{f}(w)$ is the sign of the permutation of $w$ on the fixed points of $t$. Let odd $(\lambda)$ denote the number of odd parts of the partition $\lambda$. Saxl [34] (see also [22] or [18]) proves the following decomposition of the induced character

$$
\varphi_{\mathrm{S}_{k}}^{f}:=\operatorname{Ind}_{\mathrm{C}(t)}^{\mathrm{S}_{n}}\left(\pi_{f}\right)=\sum_{\substack{\lambda \vdash k \\ \operatorname{odd}(\lambda)=f}} \chi_{\mathrm{S}_{k}}^{\lambda} \quad \text { and thus } \quad \varphi_{\mathrm{S}_{k}}:=\sum_{\ell=0}^{\lfloor k / 2\rfloor} \varphi_{\mathrm{S}_{k}}^{k-2 \ell}=\sum_{\lambda \vdash k} \chi_{\mathrm{S}_{k}}^{\lambda} .
$$

This generalizes the classic result (see [38, Theorem IV] or [19, 5.4.23]) for fixedpoint-free permutations, i.e., the case where $f=0$. In this case, there are no fixed points and $\pi_{0}$ is the trivial character of $\mathrm{C}(t)$.

The number of involutions in $\mathrm{S}_{k}$ having $f=k-2 \ell$ fixed points and $\ell$ transpositions is

$\left|I_{\mathrm{S}_{k}}^{f}\right|=\left|\mathrm{I}_{\mathrm{S}_{k}}^{k-2 \ell}\right|=\left(\begin{array}{c}k \\ 2 \ell\end{array}\right)(2 \ell-1) ! !, \quad$ where $(2 \ell-1) ! !=(2 \ell-1)(2 \ell-3) \cdots 3 \cdot 1$.

If we let $\mathrm{S}_{k}=\left|\mathrm{I}_{\mathrm{S}_{k}}\right|=\sum_{\ell=0}^{\lfloor k / 2\rfloor}\left|\mathrm{I}_{\mathrm{S}_{k}}^{k-2 \ell}\right|$ denote the total number of involutions in $\mathrm{S}_{k}$, then $\boldsymbol{S}_{k}$ is the degree of $\varphi S_{k}$ and is the sum of the dimensions of the irreducible $S_{k}$ modules. The first few values of $s_{k}$ are 1, 1, 2, 4, 10, 26, 76, 232, 764, 2620, and 9496. The sequence $s_{k}$ is [37, A000085] and has the well-known exponential generating function $e^{x^{2} / 2+x}=\sum_{k=0}^{\infty} \mathrm{s}_{k} \frac{x^{k}}{k !}$.

\subsection{The model representation of $\mathrm{S}_{k}$}

We now construct the corresponding induced model representation. For $t \in \mathrm{I}_{\mathrm{S}_{k}}^{f}$, let $\mathrm{M}_{t}=\mathbb{K} t$ be the 1-dimensional $\mathrm{C}(t)$-module with character $\pi_{f}$, so that $c \in \mathrm{C}(t)$ acts on $t$ by $c \cdot t=S(c, t) c t c^{-1}=S(c, t) t$, where $S(c, t)=\pi_{f}(c)$ is the sign of the permutation induced by $c$ on the fixed points of $t$. The cosets of $\mathrm{C}(t)$ in $\mathrm{S}_{k}$ are in bijection with the $\mathrm{S}_{k}$-orbits of $t$, which is the set of involutions $\mathrm{I}_{\mathrm{S}_{k}}^{f}$. Consider the induced module

$$
\operatorname{Ind}_{\mathrm{C}(t)}^{\mathrm{S}_{k}}\left(\mathrm{M}_{t}\right) \cong \mathbb{K S}_{k} \otimes_{\mathrm{C}(t)} t,
$$

where the $\mathrm{S}_{k}$ action is given by $x \cdot\left(w \otimes \mathrm{C}_{(t)} t\right)=x w \otimes \mathrm{C}(t) t$, for all $w, x \in \mathrm{S}_{k}$, and is extended linearly to $\mathbb{K} \mathrm{S}_{k} \otimes{ }_{\mathrm{C}(t)} \mathrm{M}_{t}$. Since $\mathrm{M}_{t}$ is 1-dimensional, $\operatorname{dim}\left(\operatorname{Ind}_{\mathrm{C}(t)} \mathrm{S}_{k}\left(\mathrm{M}_{t}\right)\right)=$ 
$\left|\mathrm{S}_{k}\right| /|\mathbf{C}(t)|=\left|\mathrm{I}_{\mathrm{S}_{k}}^{f}\right|$. Let $\left\{w_{s} \mid s \in \mathrm{I}_{\mathrm{S}_{k}}^{f}\right\}$ be a set of distinct coset representatives of $\mathbf{C}(t) \in \mathrm{S}_{k}$ such that $w_{s} t w_{s}^{-1}=s$. If $w \in \mathrm{S}_{k}$ with $w=w_{s} c \in w_{s} \mathbf{C}(t)$, then since the tensor product is over $\mathrm{C}(t)$, we have $w \otimes \mathrm{C}_{(t)} t=w_{s} c \otimes \mathrm{C}(t)_{t} t=w_{s} \otimes_{\mathrm{C}(t)} c \cdot t=$ $S(c, t) w_{s} \otimes \mathrm{C}_{(t)} t$. Thus, the vectors of the form $w_{s} \otimes_{\mathrm{C}(t)} t \operatorname{span} \operatorname{Ind}_{\mathrm{C}(t)}^{\mathrm{S}_{k}}\left(\mathrm{M}_{t}\right)$ and by comparing dimensions $\left\{w_{s} \otimes \mathrm{C}(t) t \mid s \in \mathrm{I}_{\mathrm{S}_{k}}^{f}\right\}$ are a basis of $\operatorname{Ind}_{\mathrm{C}(t)} \mathrm{S}_{k}\left(\mathrm{M}_{t}\right)$.

The induced module $\operatorname{Ind}_{\mathrm{C}(t)}^{\mathrm{S}_{k}}\left(\mathrm{M}_{t}\right)$ has a nice combinatorial construction. If $w \in \mathrm{S}_{k}$ and $t \in \mathrm{I}_{\mathrm{S}_{k}}^{f}$ then $w t w^{-1} \in \mathrm{I}_{\mathrm{S}_{k}}^{f}$ is an involution with the same number $f$ of fixed points as $t$. However, the relative position of the fixed points is permuted in the map $t \mapsto w t w^{-1}$. Define $S(w, t)$ to be the sign of the permutation induced on the fixed points of $t$ under conjugation. That is,

$$
S(w, t)=(-1)^{\mid\{1 \leq i<j \leq k \mid t(i)=i, t(j)=j, \text { and } w(i)>w(j)\} \mid} .
$$

For example, when the following involution is conjugated,

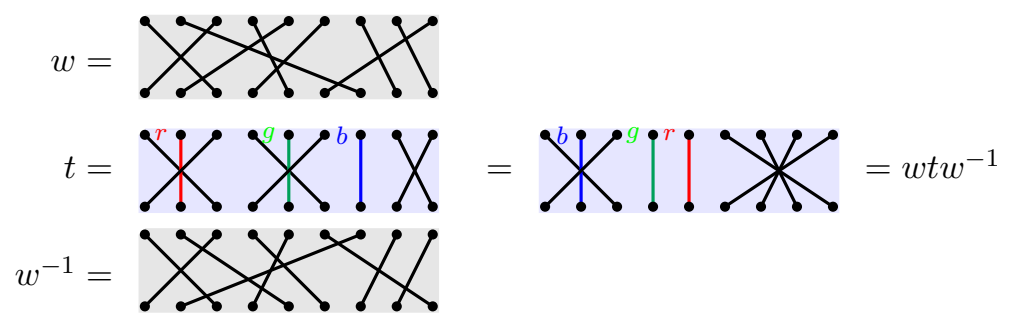

the three fixed points $(r, g, b)$ are permuted to $(b, g, r)$ which is an induced permutation of sign -1 .

Inside of the group algebra $\mathbb{K} S_{k}$ define

$$
\mathrm{M}_{\mathrm{S}_{k}}^{f}=\mathbb{K}-\operatorname{span}\left\{t \mid t \in \mathrm{I}_{\mathrm{S}_{k}}^{f}\right\}
$$

Define an action of $w \in \mathrm{S}_{k}$ on a basis element $t \in \mathrm{I}_{\mathrm{S}_{k}}^{f}$ by

$$
w \cdot t=S(w, t) w t w^{-1}
$$

which we refer to as signed conjugation. Let $\mathrm{S}_{k}$ act on $\mathrm{M}_{\mathrm{S}_{k}}^{f}$ by extending the action of (3.7) linearly.

Proposition 3.1 For $f=k-2 \ell$ with $0 \leq \ell \leq\lfloor k / 2\rfloor$, we have $\mathrm{M}_{\mathrm{S}_{k}}^{f} \cong \operatorname{Ind}_{\mathrm{C}(t)}^{\mathrm{S}_{k}}\left(\mathrm{M}_{t}\right)$.

Proof Let $s_{i}=(i, i+1)$ denote the simple transposition (given here in cycle notation) that exchanges $i$ and $i+1$. Then $s_{1}, \ldots, s_{k-1}$ generate $S_{k}$, and the length $\ell(w)$ of $w \in \mathbf{S}_{k}$ is the minimum number of simple transpositions needed to write $w$ as a product of simple transpositions. Consider the coset $w \mathbf{C}(t)$ in $\mathrm{S}_{k}$ and let $w t w^{-1}=s$. We claim that if $w$ is of minimal length among all permutations in $w \mathbf{C}(t)$, then under the map $t \mapsto w t w^{-1}=s$ the relative position of the the fixed points of $t$ is not 
changed. This can be readily verified from the diagram calculus: the length $\ell(w)$ is the number of crossings in the permutation diagram of $w$, and thus the permutation of minimal length that conjugates $t$ to $s$ does not exchange any of the fixed points of $t$. Now, for $s \in \mathrm{I}_{\mathrm{S}_{k}}^{f}$ let $w_{s}$ be the unique minimal length coset representative such that $w_{s} t w_{s}^{-1}=s$. Then $\left\{w_{s} \mid s \in \mathrm{I}_{\mathrm{S}_{k}}^{f}\right\}$ is a set of distinct coset representatives of $\mathrm{C}(t) \in \mathrm{S}_{k}$.

We now show that $x \in \mathrm{S}_{k}$ acts on the basis $\left\{w_{s} \otimes \mathrm{C}(t) t \mid s \in \mathrm{I}_{\mathrm{S}_{k}}^{f}\right\}$ of $\operatorname{Ind}_{\mathrm{C}(t)} \mathrm{S}_{k}\left(\mathrm{M}_{t}\right)$ identically to the way that $x$ acts on the basis $\left\{s \in \mathrm{I}_{\mathrm{S}_{k}}^{f}\right\}$ of $\mathrm{M}_{\mathrm{S}_{k}}^{f}$. We know that $x w_{s} \in w_{r} \mathrm{C}(t)$ for some $r \in \mathrm{I}_{\mathrm{S}_{k}}^{f}$ so $x w_{s}=w_{r} c$ for $c \in \mathrm{C}(t)$, and thus

$x \cdot\left(w_{s} \otimes_{\mathrm{C}(t)} t\right)=x w_{s} \otimes_{\mathrm{C}(t)} t=w_{r} c \otimes_{\mathrm{C}(t)} t=w_{r} \otimes_{\mathrm{C}(t)} c \cdot t=S(c, t)\left(w_{r} \otimes_{\mathrm{C}(t)} t\right)$.

Now observe that $x=w_{r} c w_{s}^{-1}$ so

$$
x s x^{-1}=\left(w_{r} c w_{s}^{-1}\right)\left(w_{s} t w_{s}^{-1}\right)\left(w_{s} c^{-1} w_{r}^{-1}\right)=w_{r}\left(c t c^{-1}\right) w_{r}^{-1}=w_{r} t w_{r}^{-1}=r .
$$

Furthermore, since $w_{r}$ does not change the relative order of the fixed points of $t$, the only place where the relative order of the fixed points of $t$ was changed was in the conjugation $c t c^{-1}=t$. Thus $S(x, s)=S(c, t)$ and so $x \cdot s=S(x, s) x s x^{-1}$.

Now, let $\mathbb{K}$ be chosen so that $\mathbb{K} S_{k}$ is semisimple (for example, $\operatorname{char}(\mathbb{K})=0$ ), and define

$$
\mathrm{M}_{\mathrm{S}_{k}}=\mathbb{K} \text {-span }\left\{t \mid t \in \mathrm{I}_{k}\right\}=\bigoplus_{\ell=0}^{\lfloor n / 2\rfloor} \mathrm{M}_{\mathrm{S}_{k}}^{k-2 \ell}
$$

Let $\Lambda_{\mathrm{S}_{k}}^{f}=\{\lambda \vdash k \mid \operatorname{odd}(\lambda)=f\}$. Applying Proposition 3.1 to (3.8) and using (3.2) gives

Theorem 3.1 Under signed conjugation (4.2), $\mathrm{M}_{\mathrm{S}_{k}}$ decomposes into irreducible $\mathrm{S}_{k}$ modules as

$$
\mathrm{M}_{\mathrm{S}_{k}}=\bigoplus_{\ell=0}^{\lfloor n / 2\rfloor} \mathrm{M}_{\mathrm{S}_{k}}^{k-2 \ell} \cong \bigoplus_{\ell=0}^{\lfloor n / 2\rfloor} \bigoplus_{\lambda \in \Lambda_{\mathrm{S}_{k}}^{k-2 \ell}} \mathrm{S}_{k}^{\lambda}=\bigoplus_{f=0}^{k} \bigoplus_{\lambda \in \Lambda_{\mathrm{S}_{k}}^{f}} \mathrm{~S}_{k}^{\lambda}=\bigoplus_{\lambda \vdash n} \mathrm{~S}_{k}^{\lambda}
$$

where $\Lambda_{\mathrm{S}_{k}}^{f}=\emptyset$ if there are no partitions of $k$ with $f$ odd parts.

\subsection{Comparison with other Gelfand models for $\mathrm{S}_{k}$}

Adin, Postnikov, and Roichman [1] (and also [23]) study a slightly different combinatorial models for $S_{k}$, and it is the analog of this model that Mazorchuk derives for the diagram algebras in [27]. The sign in [1] is computed on the two cycles of $t \in \mathrm{I}_{k}^{f}$ as follows:

$$
s(w, t)=(-1)^{\mid\{1 \leq i<j \leq k \mid t(i)=j, t(j)=i \text {, and } w(i)>w(j)\} \mid} .
$$


and the action of $\mathrm{S}_{k}$ on $\mathrm{I}_{k}^{f}$ is given as

$$
w \cdot t=s(w, t) w t w^{-1}, \quad \text { for } w \in S_{k} \text { and } t \in \mathrm{I}_{k}^{f} .
$$

We let $\overline{\mathrm{M}}_{k}^{f}$ denote the corresponding $\mathrm{S}_{k}$ module, and let $\overline{\mathrm{M}}_{k}=\oplus_{\ell=0}^{\lfloor k / 2\rfloor} \overline{\mathrm{M}}_{k}^{k-2 \ell}$. In [1] it is proved that $\overline{\mathrm{M}}_{k}$ is a Gelfand model for $\mathrm{S}_{k}$. Moreover, the action is given a $q$-extension in [1] to a Gelfand model for the Iwahori-Hecke algebra $\mathrm{H}_{k}(q)$ of $\mathrm{S}_{k}$. In what follows we prove that the Adin-Postnikov-Roichman model differs from the Saxl model by the sign representation.

Proposition 3.2 For each $0 \leq f \leq k$ such that $k-f$ is even, we have $\mathrm{M}_{\mathrm{S}_{k}}^{f} \cong$ $\overline{\mathrm{M}}_{\mathrm{S}_{k}}^{f} \otimes \mathrm{S}_{k}^{\mathrm{sign}}$, where $\mathrm{S}_{k}^{\text {sign }}$ is the sign representation of $\mathrm{S}_{k}$.

Proof Let $t \in \mathrm{I}_{\mathrm{S}_{k}}^{f}$ and let $w \in \mathrm{S}_{k}$ such that $w t w^{-1}=t$. That is, the $t$ - $t$ entry of the matrix of $w$ is nonzero (in both $\overline{\mathrm{M}}_{\mathrm{S}_{k}}^{f}$ and $\mathrm{M}_{\mathrm{S}_{k}}^{f}$ ) and thus contributes to the trace. Let $F$ be the set of fixed points of $t$ and let $t=\left(a_{1}, b_{1}\right)\left(a_{2}, b_{2}\right) \cdots\left(a_{\ell}, b_{\ell}\right)$ be the decomposition of $t$ into $\ell=(k-f) / 2$ disjoint 2-cycles with $a_{i}<b_{i}$ for each $i$. Thus the complement of $F$ in $\{1,2, \ldots, k\}$ is $\bar{F}=\left\{a_{1}, b_{1}, a_{2}, b_{2}, \ldots, a_{\ell}, b_{\ell}\right\}$.

Since $w t w^{-1}=t$, we know that $w$ permutes the fixed points $F$ of $t$. Furthermore, $w$ permutes the transpositions among themselves and possibly transposes the endpoints of the transpositions. We factor $w$ according to these three actions. Let $w_{a}, w_{b}, w_{\pi} \in$ $\mathrm{S}_{k}$ be defined as follows:

1. $w_{b}(c)=c$ if $c \in \bar{F}$ and $w_{b}(d)=w(d)$ if $d \in F$; thus, $w_{b}$ permutes the fixed points of $t$ as in $w$ and fixes the others.

2. $w_{a}(d)=d$ if $d \in F, w_{a}\left(a_{i}\right)=b_{i}$ and $w_{a}\left(b_{i}\right)=a_{i}$ if $w\left(a_{i}\right)>w\left(b_{i}\right)$, and $w_{a}\left(a_{i}\right)=a_{i}$ and $w_{a}\left(b_{i}\right)=b_{i}$ if $w\left(a_{i}\right)<w\left(b_{i}\right)$; thus $w_{a}$ transposes the endpoints of the transpositions in $t$ if they are transposed by $w$.

3. $w_{\pi}(d)=d$ if $d \in F$ and $w_{\pi}\left(a_{i}\right)=a_{\pi(i)}$ and $w_{\pi}\left(b_{i}\right)=b_{\pi(i)}$ where $\pi$ is the permutation on the transpositions induced by $w$.

It is easy to check, by examining the values of these permutations on each element of $F \cup \bar{F}=\{1, \ldots, k\}$, that

$$
w=w_{\pi} w_{a} w_{b}, \quad \text { and thus } \operatorname{sign}(w)=\operatorname{sign}\left(w_{\pi}\right) \operatorname{sign}\left(w_{a}\right) \operatorname{sign}\left(w_{b}\right) .
$$

Furthermore, by the definition of $w_{a}, w_{b}, S(w, t)$, and $s(w, t)$ we have $\operatorname{sign}\left(w_{b}\right)=$ $S(w, t)$ and $\operatorname{sign}\left(w_{a}\right)=s(w, t)$. Finally, since $w_{\pi}$ permutes the set of transpositions $\left(a_{i}, b_{i}\right)$, keeping $a_{i}<b_{i}$, it can be written as a product of pairs of transpositions of the form $\left(a_{i}, a_{j}\right)\left(b_{i}, b_{j}\right)$. Thus, $\operatorname{sign}\left(w_{\pi}\right)=1$, and we have $\operatorname{sign}(w)=S(w, t) s(w, t)$ or, equivalently, $S(w, t)=s(w, t) \operatorname{sign}(w)$, for each $t$ such that $w t w^{-1}=t$. It follows that the characters of $w$ on $\mathbf{M}_{\mathbf{S}_{k}}^{f}$ and $\overline{\mathbf{M}}_{\mathbf{S}_{k}}^{f} \otimes \mathrm{S}_{k}^{\text {sign }}$ are equal and the modules are isomorphic. 


\section{Gelfand models from the jones basic construction}

In this section, we show how to construct model representations for a tower of algebras $\left(A_{k}\right)_{k \geq 0}$ that is obtained by repeated Jones basic constructions $([10,11,20])$ from a tower of algebras $\left(\mathrm{C}_{k}\right)_{k \geq 0}$ for which we already have a model representation. Each of the diagram algebras of interest in this paper has such an inductive structure with $\mathrm{C}_{k} \cong \mathbb{K} \mathrm{S}_{k}$, in the case of the nonplanar algebras, and $\mathrm{C}_{k} \cong \mathbb{K} \mathbf{1}$, in the case of the planar algebras. We lift Saxl's model for $S_{k}$ in the first case and the trivial model in the second.

\subsection{The Jones basic construction}

Let $A_{0} \subseteq A_{1} \subseteq A_{2} \subseteq \cdots$ be a tower of inclusions of finite-dimensional, semisimple algebras with 1 over the algebraically closed field $\mathbb{K}$. Assume that there exist elements $0 \neq \mathrm{e}_{k} \in \mathrm{A}_{k+1}$, and $k^{\prime}<k$, which satisfy the following relations

(a) $\mathrm{e}_{k}^{2}=\mathrm{e}_{k}$,

(b) $\mathrm{e}_{k} a=a \mathrm{e}_{k}$, for all $a \in \mathrm{A}_{k^{\prime}}$,

(c) $\mathrm{A}_{k^{\prime}} \cong \mathrm{A}_{k^{\prime}} \mathrm{e}_{k} \cong \mathrm{e}_{k} \mathrm{~A}_{k} \mathrm{e}_{k}$ via the map $a \mapsto a \mathrm{e}_{k}$ for all $a \in \mathrm{A}_{k}$.

In the examples in this paper $k^{\prime}=k-1$ or $k^{\prime}=k-2$. Define the map $\varepsilon_{k}: \mathrm{A}_{k} \rightarrow \mathrm{A}_{k^{\prime}}$, called the conditional expectation, such that $\varepsilon_{k}(b)$ is the unique element in $A_{k^{\prime}}$ such that $\mathrm{e}_{k} b \mathrm{e}_{k}=\varepsilon_{k}(b) \mathrm{e}_{k}$. Let $A_{k} \mathrm{e}_{k}$ be a module for $\mathrm{A}_{k} \mathrm{e}_{k} \mathrm{~A}_{k}$ by multiplication on the left and a module for $\mathrm{e}_{k} \mathrm{~A}_{k} \mathrm{e}_{k}$ by multiplication on the right. Wenzl [39] proves the following (see also [13, Theorem 2.6], [14, Prop. 5.1.3]),

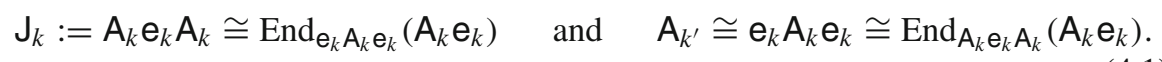

Since $J_{k}=A_{k} e_{k} A_{k} \cong \operatorname{End}_{e_{k}} A_{k} e_{k}\left(A_{k} e_{k}\right)$ it is an ideal and a semisimple subalgebra (with unit $\mathrm{e}_{k}$ ). Thus, there exists a subalgebra $\mathrm{C}_{k} \subseteq \mathrm{A}_{k}$ such that

$$
\mathrm{A}_{k}=\mathrm{J}_{k} \oplus \mathrm{C}_{k} \quad \text { and } \quad \mathrm{A}_{k} / \mathrm{J}_{k} \cong \mathrm{C}_{k}
$$

Let $\Lambda_{\mathrm{A}_{k}}$ index the irreducible $\mathrm{A}_{k}$-modules. It follows (from double centralizer theory) that the irreducible modules for $A_{k^{\prime}}$ and $J_{k}$ are indexed by the same set $\Lambda_{A_{k^{\prime}}}$. Thus by (4.2), we have

$$
\Lambda_{\mathrm{A}_{k}}=\Lambda_{\mathrm{J}_{k}} \sqcup \Lambda_{\mathrm{C}_{k}}=\Lambda_{\mathrm{A}_{k^{\prime}}} \sqcup \Lambda_{\mathrm{C}_{k}}
$$

Applying (4.3) recursively gives $\Lambda_{\mathrm{A}_{k}}=\Lambda_{\mathrm{C}_{0}} \sqcup \Lambda_{\mathrm{C}_{1}} \sqcup \Lambda_{\mathrm{C}_{2}} \sqcup \cdots \sqcup \Lambda_{\mathrm{C}_{k}}$, where for some values of $i$, we may have $\mathrm{C}_{i}=0$ and $\Lambda_{\mathrm{C}_{i}}=\emptyset$ (see the Examples in Sect. 5).

If $\chi$ is any character of $A_{k}$, then by (4.2), $\chi$ is completely determined by its values on $\mathrm{J}_{k}$ and $\mathrm{C}_{k}$. If $a \in \mathrm{J}_{k}=\mathrm{A}_{k} \mathrm{e}_{k} \mathrm{~A}_{k}$, then $a=a_{1} \mathrm{e}_{k} a_{2}$ for $a_{1}, a_{2} \in \mathrm{A}_{k}$, and by the trace property of $\chi$,

$$
\chi(a)=\chi\left(a_{1} \mathrm{e}_{k} a_{2}\right)=\chi\left(a_{2} a_{1} \mathrm{e}_{k}\right)=\chi\left(a_{2} a_{1} \mathrm{e}_{k}^{2}\right)=\chi\left(\mathrm{e}_{k} a_{2} a_{1} \mathrm{e}_{k}\right)=\chi\left(\varepsilon\left(a_{2} a_{1}\right) \mathrm{e}_{k}\right) .
$$


Thus, character values on $\mathrm{J}_{k}$ are completely determined by their values on $a \mathrm{e}_{k}$ for $a \in \mathrm{A}_{k^{\prime}}$, and

Characters of $\mathrm{A}_{k}$ are completely determined by their values on $b \in \mathrm{C}_{k}$ and $a \mathrm{e}_{k}$, for $a \in \mathrm{A}_{k^{\prime}}$.

The following result is proved in [13] and [14] for algebras $A_{k}$ satisfying (a), (b), (c) above with quotient $\mathrm{C}_{k}$ defined as in (4.2). If $\lambda \in \Lambda_{\mathrm{A}_{k}}$, then

$$
\chi_{\mathrm{A}_{k}}^{\lambda}(a)= \begin{cases}\chi_{\mathrm{C}_{k}}^{\lambda}(a), & \text { if } \lambda \in \Lambda_{\mathrm{C}_{k}} \text { and } a \in \mathrm{C}_{k}, \\ 0, & \text { if } \lambda \in \Lambda_{\mathrm{C}_{k}} \text { and } a \in \mathrm{J}_{k}, \\ \chi_{\mathrm{A}_{k^{\prime}}}^{\lambda}\left(a^{\prime}\right), & \text { if } \lambda \in \Lambda_{\mathrm{A}_{k^{\prime}}} \text { and } a=a^{\prime} \mathrm{e}_{k} \text { with } a^{\prime} \in \mathrm{A}_{k^{\prime}} .\end{cases}
$$

The character values, $\chi_{\mathrm{A}_{k}}^{\lambda}(a)$ for $\lambda \in \Lambda_{\mathrm{A}_{k^{\prime}}}$ and $a \in \mathrm{C}_{k}$, are harder to compute but not needed here.

Now assume that we have a model representation $\mathrm{M}_{\mathrm{C}_{r}}$ of $\mathrm{C}_{r}$, for each $0 \leq r \leq k$, with corresponding character $\varphi_{\mathrm{C}_{k}}$. Thus,

$$
\mathrm{M}_{\mathrm{C}_{r}} \cong \bigoplus_{\lambda \in \Lambda_{\mathrm{C}_{r}}} \mathrm{C}_{r}^{\lambda} \quad \text { and } \quad \varphi_{\mathrm{C}_{r}}=\sum_{\lambda \in \Lambda_{\mathrm{C}_{r}}} \chi_{\mathrm{C}_{r}}^{\lambda}
$$

where $\left\{\mathrm{C}_{r}^{\lambda} \mid \lambda \in \Lambda_{\mathrm{C}_{r}}\right\}$ is the set of the irreducible $\mathrm{C}_{r}$-modules with characters $\chi_{\mathrm{C}_{r}}^{\lambda}, \lambda \in$ $\Lambda_{\mathrm{C}_{r}}$. Suppose further that we have constructed a module $\mathrm{M}_{\mathrm{A}_{k}}$ for $\mathrm{A}_{k}$ which decomposes into $\mathrm{A}_{k}$-submodules $\mathrm{M}_{\mathrm{A}_{k}}=\bigoplus_{r=0}^{k} \mathrm{M}_{\mathrm{A}_{k}}^{r}$ satisfying

(M1) $\mathrm{M}_{\mathrm{A}_{k}}^{r}$ and $\mathrm{M}_{\mathrm{A}_{k}}^{s}$ have no common irreducible constituents if $r \neq s$, and (M2) The character $\varphi_{\mathrm{A}_{k}}^{r}$ of $\mathrm{M}_{\mathrm{A}_{k}}^{r}$ satisfies

$$
\varphi_{\mathrm{A}_{k}}^{r}(a)= \begin{cases}\varphi_{\mathrm{C}_{k}}(a), & \text { if } r=k \text { and } a \in \mathrm{C}_{k}, \\ 0, & \text { if } r=k \text { and } a \in \mathrm{J}_{k}, \\ \varphi_{\mathrm{A}_{k}^{\prime}}^{r}\left(a^{\prime}\right), & \text { if } r<k \text { and } a=a^{\prime} \mathrm{e}_{k} \text { with } a^{\prime} \in \mathrm{A}_{k-1} .\end{cases}
$$

Then the following theorem tells us that $M_{A_{k}}$ is a model for $A_{k}$.

Theorem 4.1 Let $\mathrm{A}_{0} \subseteq \mathrm{A}_{1} \subseteq \ldots \subseteq \mathrm{A}_{k}$ be a tower of semisimple algebras obtained by Jones basic constructions from $\overline{\mathrm{C}}_{0} \subseteq \mathrm{C}_{1} \subseteq \ldots \subseteq \mathrm{C}_{k}$. If $\mathrm{M}_{k}=\bigoplus_{r=0}^{k} \mathrm{M}_{\mathrm{A}_{k}}^{r}$ is an $\mathrm{A}_{k}$ module satisfying (M1) and (M2), then

$$
\mathrm{M}_{\mathrm{A}_{k}}^{r}=\bigoplus_{\lambda \in \Lambda_{\mathrm{C}_{r}}} \mathrm{~A}_{k}^{\lambda}, \quad \text { and thus } \quad \mathrm{M}_{\mathrm{A}_{k}}=\bigoplus_{r=0}^{k} \mathrm{M}_{\mathrm{A}_{k}}^{r}=\bigoplus_{\lambda \in \Lambda_{\mathrm{A}_{k}}} \mathrm{M}_{\mathrm{A}_{k}}^{\lambda} \text {. }
$$

Proof We prove this on the character level; namely, we show that $\varphi_{\mathrm{A}_{k}}^{r}=\sum_{\lambda \in \Lambda_{\mathrm{C}_{r}}} \chi_{\mathrm{A}_{k}}^{\lambda}$ and $\varphi_{\mathrm{A}_{k}}=\sum_{r=0}^{k} \varphi_{\mathrm{A}_{k}}^{r}=\sum_{\lambda \in \Lambda_{\mathrm{A}_{k}}} \chi_{\mathrm{A}_{k}}^{\lambda}$ (the second statement follows immediately 
from the first). Our proof is by induction on $k$, with $k=0$ being trivial. Let $k>0$ and first consider the situation where $r=k$. Using (4.7), (4.6), and (4.5) we have

$$
\begin{array}{lr}
\varphi_{\mathrm{A}_{k}}^{k}(a)=\varphi_{\mathrm{C}_{k}}(a)=\sum_{\lambda \in \Lambda_{\mathrm{C}_{k}}} \chi_{\mathrm{C}_{k}}^{\lambda}(a)=\sum_{\lambda \in \Lambda_{\mathrm{C}_{k}}} \chi_{\mathrm{A}_{k}}^{\lambda}(a), & \text { for all } a \in \mathrm{C}_{k}, \text { and } \\
\varphi_{\mathrm{A}_{k}}^{k}(a)=0=\sum_{\lambda \in \Lambda_{\mathrm{C}_{k}}} \chi_{\mathrm{A}_{k}}^{\lambda}(a), & \text { for all } a \in \mathrm{J}_{k} .
\end{array}
$$

Since characters of $\mathrm{A}_{k}$ are completely determined by their values on $\mathrm{C}_{k}$ and $\mathrm{J}_{k}$, we have that $\varphi_{\mathrm{A}_{k}}^{k}=\sum_{\lambda \in \Lambda_{\mathrm{C}_{k}}} \chi_{\mathrm{A}_{k}}^{\lambda}$ is the decomposition of $\varphi_{\mathrm{A}_{k}}^{k}$ into irreducible characters.

Now let $r<k$. The previous paragraph and (M1) tell us that the decomposition of $\varphi_{\mathrm{A}_{k}}^{r}$ into irreducibles does not involve any $\chi_{\mathrm{A}_{k}}^{\lambda}$ with $\lambda \in \Lambda_{\mathrm{C}_{k}}$. Thus by (4.3) it is of the form

$$
\varphi_{\mathrm{A}_{k}}^{r}=\sum_{\lambda \in \Lambda_{\mathrm{A}_{k^{\prime}}}} a_{\lambda} \chi_{\mathrm{A}_{k}}^{\lambda}, \quad \text { for some } a_{\lambda} \in \mathbb{Z}_{\geq 0}
$$

Let $a=a^{\prime} \mathrm{e}_{k}$ with $a^{\prime} \in \mathrm{A}_{k^{\prime}}$. For each $\lambda \in \Lambda_{\mathrm{A}_{k^{\prime}}}$ we have $\chi_{\mathrm{A}_{k}}^{\lambda}(a)=\chi_{\mathrm{A}_{k^{\prime}}}^{\lambda}\left(a^{\prime}\right)$ by (4.5), and thus $\varphi_{\mathrm{A}_{k}}^{r}(a)=\varphi_{\mathrm{A}_{k^{\prime}}}^{r}\left(a^{\prime}\right)$ by (4.8). Furthermore, we can apply the inductive hypothesis since $k^{\prime}<k$,

$$
\varphi_{\mathrm{A}_{k}}^{r}(a)=\varphi_{\mathrm{A}_{k^{\prime}}}^{r}\left(a^{\prime}\right)=\sum_{\lambda \in \Lambda_{\mathrm{C}_{r}}} \chi_{\mathrm{A}_{k^{\prime}}}^{\lambda}\left(a^{\prime}\right)=\sum_{\lambda \in \Lambda_{\mathrm{C}_{r}}} \chi_{\mathrm{A}_{k}}^{\lambda}(a) .
$$

Thus, $a_{\lambda}=1$ for $\lambda \in \Lambda_{\mathrm{C}_{r}}$ and $a_{\lambda}=0$, otherwise, as desired.

\subsection{Jones basic construction for subalgebras of the partition algebra}

Let $A_{k}$ be the partition algebra or one of its subalgebras described in Sect. 2.3 with $x \in \mathbb{K}$ chosen such that $\mathrm{A}_{k}$ is semisimple. Let $\mathcal{A}_{k}$ be the basis of diagrams which span $A_{k}$. There is a natural embedding $A_{k-1} \subseteq A_{k}$, given by placing an identity edge to the right of any diagram $d \in \mathrm{A}_{k-1}$, i.e., $\quad d \quad \mapsto \quad d \quad$ I. Let $\mathrm{J}_{k} \subseteq \mathrm{A}_{k}$ be the ideal spanned by the diagrams of $\mathrm{A}_{k}$ having rank less than $k$. Then,

$$
\mathrm{A}_{k} \cong \mathrm{J}_{k} \oplus \mathrm{C}_{k}
$$

where $\mathrm{C}_{k}$ is the span of the diagrams of rank exactly equal to $k$. In the examples of this paper,

$\mathrm{C}_{k} \cong \mathbb{K} \mathrm{S}_{k}$, when $\mathrm{A}_{k}$ is one of the nonplanar algebras $\mathrm{P}_{k}(x), \mathrm{B}_{k}(x), \mathrm{RB}_{k}(x)$ or $\mathrm{R}_{k}$, $\mathrm{C}_{k} \cong \mathbb{K} \mathbf{1}_{k}$, when $\mathrm{A}_{k}$ is one of the planar algebras $\mathrm{TL}_{k}(x), \mathrm{M}_{k}(x)$, or $\mathrm{PR}_{k}$.

Define an idempotent $\mathrm{e}_{k} \in \mathrm{J}_{k}$ by 


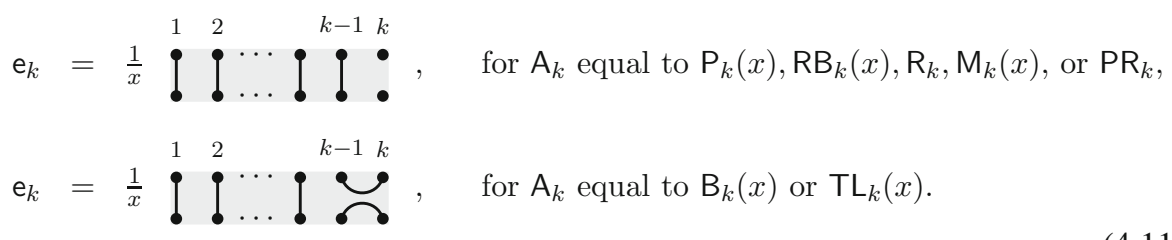

Recall that in the special cases where $\mathrm{A}_{k}=\mathrm{R}_{k}$ or $\mathrm{A}_{k}=\mathrm{PR}_{k}$ we have $x=1$. It is easy to verify by diagram multiplication that

$$
\begin{aligned}
\mathrm{J}_{k} & =\mathrm{A}_{k} \mathrm{e}_{k} \mathrm{~A}_{k}, \text { for } \mathrm{A}_{k} \text { equal to } \mathrm{P}_{k}(x), \mathrm{RB}_{k}(x), \mathrm{R}_{k}, \mathrm{M}_{k}(x), \text { or } \mathrm{PR}_{k}, \\
\mathrm{~J}_{k-1}=\mathrm{J}_{k} & =\mathrm{A}_{k} \mathrm{e}_{k} \mathrm{~A}_{k}, \text { for } \mathrm{A}_{k} \text { equal to } \mathrm{B}_{k}(x) \text { or } \mathrm{TL}_{k}(x) .
\end{aligned}
$$

Define $k^{\prime}=k-1$ or $k^{\prime}=k-2$ so that

$$
\mathrm{A}_{k^{\prime}}=\left\{\begin{array}{l}
\mathrm{A}_{k-1}, \text { if } \mathrm{A}_{k} \text { equals } \mathrm{P}_{k}(x), \mathrm{RB}_{k}(x), \mathrm{R}_{k}, \mathrm{M}_{k}(x), \text { or } \mathrm{PR}_{k}, \\
\mathrm{~A}_{k-2}, \text { if } \mathrm{A}_{k} \text { equals } \mathrm{B}_{k}(x) \text { or } \mathrm{TL}_{k}(x) .
\end{array}\right.
$$

In each of our examples it is well known, and straight-forward to verify, that $A_{k}$ satisfies properties (a), (b), (c) of the basic construction in Sect. 4.1 using the idempotent $\mathrm{e}_{k}$. Thus, $\Lambda_{\mathrm{A}_{k}}=\Lambda_{\mathrm{J}_{k-1}} \sqcup \Lambda_{\mathrm{C}_{k}}=\Lambda_{\mathrm{A}_{k}^{\prime}} \sqcup \Lambda_{\mathrm{C}_{k}}$, and by induction

$$
\begin{array}{ll}
\Lambda_{\mathrm{A}_{k}}=\bigsqcup_{r=0}^{k} \Lambda_{\mathrm{C}_{r}}, & \text { for } \mathrm{A}_{k} \text { equal to } \mathrm{P}_{k}(x), \mathrm{RB}_{k}(x), \mathrm{R}_{k}, \mathrm{M}_{k}(x), \text { or } \mathrm{PR}_{k}, \\
\Lambda_{\mathrm{A}_{k}}=\bigsqcup_{\ell=0}^{[k / 2\rfloor} \Lambda_{\mathrm{C}_{k-2 \ell}}, & \text { for } \mathrm{A}_{k} \text { equal to } \mathrm{B}_{k}(x) \text { or } \mathrm{TL}_{k}(x) .
\end{array}
$$

\subsection{Symmetric diagrams and diagram conjugation}

For any diagram $d \in \mathcal{A}_{k}$, let $d^{T} \in \mathcal{A}_{k}$ be the diagram obtained by reflecting $d$ over its horizontal axis. For example,

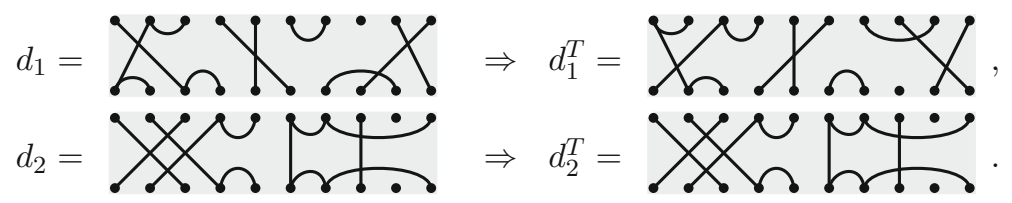

Note that the map $d \rightarrow d^{T}$ corresponds to exchanging $i \leftrightarrow i^{\prime}$ for all $i$.

We say that a diagram $d$ is symmetric if $d^{T}=d$. In our example above, $d_{2}$ is symmetric and $d_{1}$ is not. If we let $\left(i^{\prime}\right)^{\prime}=i$ and let $B^{\prime}=\left\{b^{\prime} \mid b \in B\right\}$ for a block $B$ of a partition diagram $d$, then $d$ is symmetric if it satisfies: $B \in d$ if and only if $B^{\prime} \in d$. If $d$ is a partition diagram, then we say that a block $B \in d$ is a fixed block if $B^{\prime}=B$. In our above examples, $d_{1}$ has one fixed block, $\left\{5,5^{\prime}\right\}$, and $d_{2}$ has two fixed blocks, $\left\{8,8^{\prime}\right\}$ and $\left\{6,7,10,6^{\prime}, 7^{\prime}, 10^{\prime}\right\}$. Note that 


$$
(a b)^{T}=b^{T} a^{T} \quad \text { and } \quad\left(d t d^{T}\right)^{T}=\left(d^{T}\right)^{T} t^{T} d^{T}=d t^{T} d^{T}
$$

so $t$ is symmetric if and only if $d t d^{T}$ is symmetric. We say that $d t d^{T}$ is the conjugate of $t$ by $d$. See Example 4.1. Observe that in $\mathrm{P}_{k}(x)$ the sizes of the blocks can change under conjugation.

Remark 4.1 The symmetric diagrams in the partition algebra are referred to as type$B$ set partitions in [37, A002872]. They appear in [28] and are equivalent to the horizontally symmetric $2 \times n$ letter arrays $H_{2, n}$ in [29]. They are closely related to the type- $B$ set partitions used in [31], except that in [31] the partitions are restricted to have at most one fixed block.

Remark 4.2 If we restrict our diagrams to the symmetric group, then $d^{T}$ equals $d^{-1}$, diagram conjugation corresponds to usual group conjugation, symmetric diagrams are involutions, and fixed blocks are fixed points.

Example 4.1 (a) Conjugation in the partition algebra $\mathrm{P}_{k}(x)$.

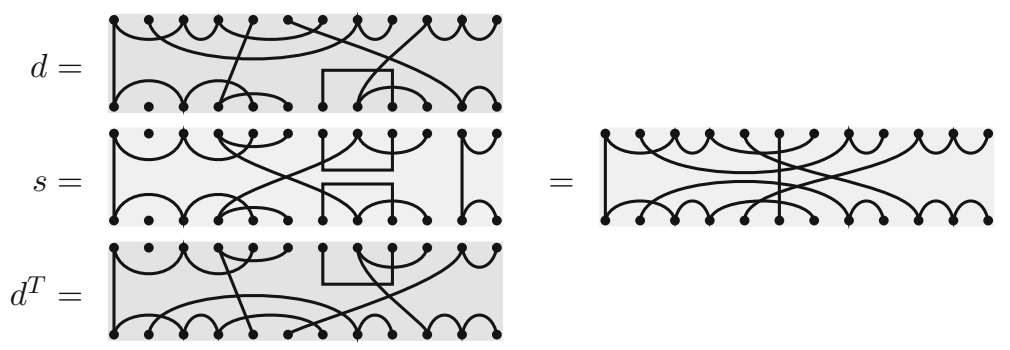

(b) Conjugation in the Brauer algebra $\mathrm{B}_{k}(x)$ :

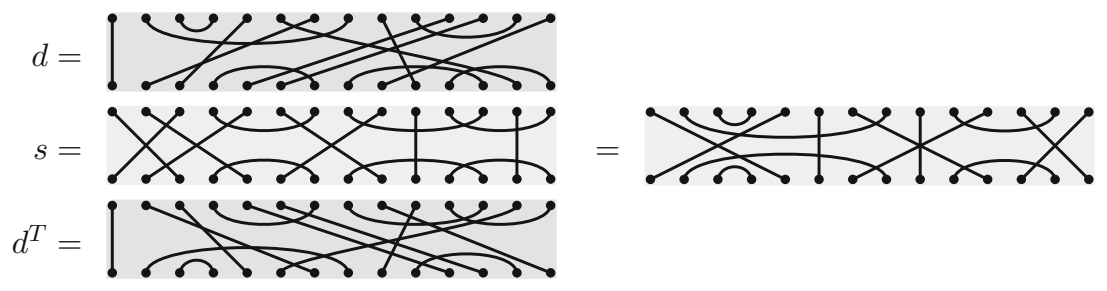

(c) Conjugation in the Temperley-Lieb algebra $\operatorname{TL}_{k}(x)$ :

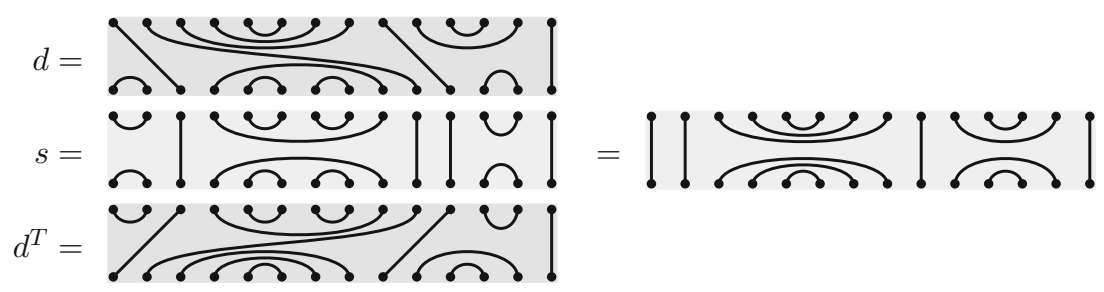




\subsection{A Gelfand model for $A_{k}$}

For any of our diagram algebras $A_{k}$, let

$$
\begin{aligned}
& \mathrm{I}_{\mathrm{A}_{k}}^{r, f}=\left\{d \in \mathcal{A}_{k} \mid d \text { is symmetric, } \operatorname{rank}(d)=r, \text { and } d \text { has } f \text { fixed blocks }\right\}, \\
& \mathrm{I}_{\mathrm{A}_{k}}^{r}=\left\{d \in \mathcal{A}_{k} \mid d \text { is symmetric, } \operatorname{rank}(d)=r\right\}, \\
& \mathrm{I}_{\mathrm{A}_{k}}=\left\{d \in \mathcal{A}_{k} \mid d \text { is symmetric }\right\} .
\end{aligned}
$$

For $0 \leq f \leq r \leq k$, define

$$
\mathrm{M}_{\mathrm{A}_{k}}^{r, f}=\mathbb{K}-\operatorname{span}\left\{d \mid d \in \mathrm{I}_{\mathrm{A}_{k}}^{r, f}\right\}
$$

where $\mathbf{M}_{\mathrm{A}_{k}}^{r, f}=0$ if $\mathrm{I}_{\mathrm{A}_{k}}^{r, f}=\emptyset$, and let

$$
\begin{aligned}
& \mathrm{M}_{\mathrm{A}_{k}}^{r}=\mathbb{K}-\operatorname{span}\left\{d \mid d \in \mathrm{I}_{\mathrm{A}_{k}}^{r}\right\}, \\
& =\bigoplus_{f=0}^{r} \mathrm{M}_{\mathrm{A}_{k}}^{r, f} \text {, } \\
& \begin{aligned}
\mathrm{M}_{\mathrm{A}_{k}} & =\mathbb{K}-\operatorname{span}\left\{d \mid d \in \mathrm{I}_{\mathrm{A}_{k}}\right\}, \\
& =\bigoplus_{r=0}^{k} \mathrm{M}_{\mathrm{A}_{k}}^{r}=\bigoplus_{r=0}^{k} \bigoplus_{f=0}^{r} \mathrm{M}_{\mathrm{A}_{k}}^{r, f} .
\end{aligned}
\end{aligned}
$$

If $d \in \mathcal{A}_{k}$ and $t \in \mathrm{I}_{\mathrm{A}_{k}}^{r, f}$, then $\operatorname{rank}\left(d \circ t \circ d^{T}\right) \leq \operatorname{rank}(t)$. When $\operatorname{rank}\left(d \circ t \circ d^{T}\right)=$ $\operatorname{rank}(t)$, the fixed blocks of $t$ have been permuted, and we let $S(d, t)$ be the sign of the permutation of the fixed blocks of $t$. For $d \in \mathcal{A}_{k}$ and $t \in \mathrm{I}_{\mathrm{A}_{k}}^{r, f}$, define

$$
d \cdot t= \begin{cases}x^{\kappa(d, t)} S(d, t) d \circ t \circ d^{T}, & \text { if } \operatorname{rank}\left(d \circ t \circ d^{T}\right)=\operatorname{rank}(t), \\ 0, & \text { if } \operatorname{rank}\left(d \circ t \circ d^{T}\right)<\operatorname{rank}(t),\end{cases}
$$

where $\kappa(d, t)$ is the number of blocks (2.1) removed from the middle row in creating $d \circ t$. We refer to the above action as signed conjugation of $t$ by $d$.

Example 4.2 (Signed Conjugation) In the following example, there is one block removed in $d \circ t$ yielding a multiple of $x$. Furthermore, the three fixed blocks of $t$ are permuted as $\left(B_{1}, B_{2}, B_{3}\right) \mapsto\left(B_{3}, B_{2}, B_{1}\right)$. Hence, the sign is $S(d, t)=-1$.

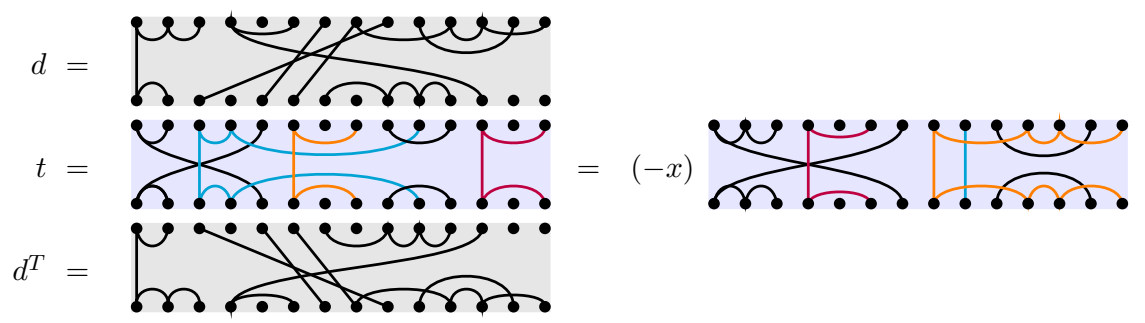

Example 4.3 The signs in Example 4.1 are (a) $x^{2}$, (b) $-x^{3}$, and (c) $x^{4}$, respectively. 
Remark 4.3 Observe the following subtlety in the definition of this action: as a product in $\mathrm{A}_{k}$ we have $d t d^{T}=x^{2 \kappa(d, t)} d \circ t \circ d^{T}$, since each block removed from the middle row in $d \circ t$ has a mirror image in $t \circ d^{T}$; however, we require $d \cdot t=x^{\kappa(d, t)} S(d, t) d \circ t \circ d^{T}$ in order to make this an algebra action, as will be seen in the proof of Proposition 4.1.

Remark 4.4 When the action in (4.18) is restricted to the symmetric group, we exactly get the action of $S_{k}$ on involutions $I_{k}$ defined in equation (3.7)

Proposition 4.1 The action defined in (4.18) makes $\mathrm{M}_{\mathrm{A}_{k}}^{r, f}$ an $\mathrm{A}_{k}$-module.

Proof We show that $\left(d_{1} d_{2}\right) \cdot t=d_{1} \cdot\left(d_{2} \cdot t\right)$. If $\operatorname{rank}\left(d \circ t \circ d^{T}\right)<\operatorname{rank}(t)$, then by the associativity of diagram multiplication we have $\left(d_{1} d_{2}\right) \cdot t=0=d_{1} \cdot\left(d_{2} \cdot t\right)$. So we assume that $\operatorname{rank}\left(d \circ t \circ d^{T}\right)=\operatorname{rank}(t)$. Let $d_{1} \circ d_{2}=d_{3}$ and let $d_{2} \circ t \circ d_{2}^{T}=s$ for some $s \in \mathrm{I}_{\mathrm{A}_{k}}^{r, f}$. Then we have,

$$
\begin{aligned}
d_{1} \cdot\left(d_{2} \cdot t\right) & =x^{\kappa\left(d_{2}, t\right)} S\left(d_{2}, t\right) d_{1} \cdot\left(d_{2} \circ t \circ d_{2}^{T}\right) \\
& =x^{\kappa\left(d_{1}, s\right)} x^{\kappa\left(d_{2}, t\right)} S\left(d_{1}, s\right) S\left(d_{2}, t\right)\left(d_{1} \circ\left(d_{2} \circ t \circ d_{2}^{T}\right) \circ d_{1}^{T}\right) \\
& =x^{\kappa\left(d_{1}, s\right)} x^{\kappa\left(d_{2}, t\right)} S\left(d_{1}, s\right) S\left(d_{2}, t\right)\left(\left(d_{1} d_{2}\right) \circ t \circ\left(d_{1} \circ d_{2}\right)^{T}\right) \\
& =x^{\kappa\left(d_{1}, s\right)} x^{\kappa\left(d_{2}, t\right)} S\left(d_{1}, s\right) S\left(d_{2}, t\right)\left(d_{3} \circ t \circ d_{3}^{T}\right) .
\end{aligned}
$$

On the other hand, $\left(d_{1} d_{2}\right) \cdot t=x^{\kappa\left(d_{1}, d_{2}\right)} d_{3} \cdot t=x^{\kappa\left(d_{1}, d_{2}\right)} x^{\kappa\left(d_{3}, t\right)} S\left(d_{3}, t\right) d_{3} t d_{3}^{T}$, so it suffices to show that

$$
S\left(d_{3}, t\right)=S\left(d_{1}, s\right) S\left(d_{2}, t\right) \quad \text { and } \quad x^{\kappa\left(d_{1}, d_{2}\right)} x^{\kappa\left(d_{3}, t\right)}=x^{\kappa\left(d_{1}, s\right)} x^{\kappa\left(d_{2}, t\right)} .
$$

From the diagram calculus, we have that $\kappa\left(d_{1}, d_{2}\right)=\kappa\left(d_{1}, s\right)$ and $\kappa\left(d_{3}, t\right)=\kappa\left(d_{2}, t\right)$, so the second equality follows immediately. The first equality corresponds to the composition of permutations of fixed blocks, and the result follows from the symmetric group fact that the sign of a permutation is multiplicative.

For $d \in \mathcal{A}_{k}$, let $\tau(d)$ be the set partition of $\{1, \ldots, k\}$ given by restricting $d$ to $\{1, \ldots, k\}$ and let $\beta(d)$ be the set partition of $\left\{1^{\prime}, \ldots, k^{\prime}\right\}$ given by restricting $d$ to $\left\{1^{\prime}, \ldots, k^{\prime}\right\}$. Thus if $t$ is symmetric, then $i \leftrightarrow i^{\prime}$ is a bijection between $\tau(t)$ and $\beta(t)$. For each $t \in \mathrm{I}_{\mathrm{A}_{k}}^{r, f}$, let $p_{t} \in \mathcal{A}_{k}$ be the unique diagram such that

(a) $\tau\left(p_{t}\right)=\tau(t)$ and $\beta\left(p_{t}\right)=\beta(t)$

(b) A block of $t$ is propagating if and only if the corresponding block of $p_{t}$ is an identity block.

For example,

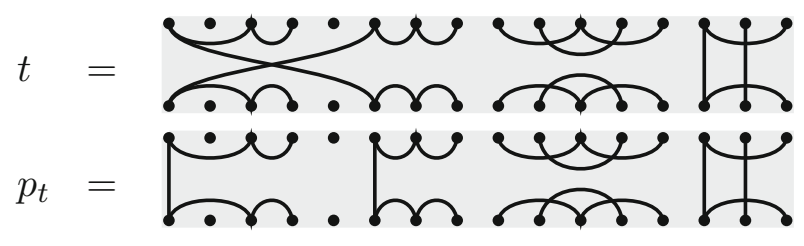


Note that $\operatorname{rank}\left(p_{t}\right)=\operatorname{rank}(t)$. It follows from this construction that

$$
p_{t} t=t p_{t}=x^{\ell} t
$$

where $\ell$ is the number of non propagating blocks of $t$. These diagrams are used in the proof of the following proposition.

Proposition 4.2 If $r \neq s$, there is no submodule of $\mathrm{M}_{\mathrm{A}_{k}}^{r, f}$ isomorphic to a submodule of $\mathrm{M}_{\mathrm{A}_{k}}^{s, g}$.

Proof By Schur's lemma, $\mathbf{M}_{\mathbf{A}_{k}}^{r, f}$ and $\mathbf{M}_{\mathbf{A}_{k}}^{s, g}$ have an isomorphic submodule if and only if there is a nontrivial $\mathrm{A}_{k}$-module homomorphism $\phi: \mathrm{M}_{\mathrm{A}_{k}}^{r, f} \rightarrow \mathrm{M}_{\mathrm{A}_{k}}^{s, g}$. Assume $r<s$, without loss of generality, and let $t \in \mathrm{I}_{\mathrm{A}_{k}}^{r, f}$. Suppose $\phi: \mathrm{M}_{\mathrm{A}_{k}}^{r, f} \rightarrow \mathrm{M}_{\mathrm{A}_{k}}^{s, g}$ is a nontrivial $A_{k}$-module homomorphism. Then by (4.19) and the fact that $\phi$ is an $A_{k}$-module homomorphism, $\phi(t)=\phi\left(x^{-\ell} p_{t} t\right)=x^{-\ell} p_{t} \phi(t)$. Now, $\phi(t)$ is a linear combination of symmetric diagrams of rank $s$, but $\operatorname{rank}\left(p_{t}\right)=\operatorname{rank}(t)<s$, and thus by (4.18), $p_{t}$ acts as 0 on each diagram in the linear combination $\phi(t)$. Thus $\phi(t)=0$ for each $t$, and there are no nontrivial $\mathrm{A}_{k}$-module homomorphisms.

Let $\varphi_{\mathrm{A}_{k}}^{r, f}$ be the character of the $\mathrm{A}_{k}$-module $\mathbf{M}_{\mathrm{A}_{k}}^{r, f}$. Then $\varphi_{\mathrm{A}_{k}}^{r}=\sum_{f=0}^{r} \varphi_{\mathrm{A}_{k}}^{r, f}$ is the character of $\mathrm{M}_{\mathrm{A}_{k}}^{r}$ and $\varphi_{\mathrm{A}_{k}}=\sum_{r=0}^{k} \varphi_{\mathrm{A}_{k}}^{r}$ is the character of $\mathrm{M}_{\mathrm{A}_{k}}$. Let $\varphi_{\mathrm{C}_{k}}^{f}$ be the restriction of $\varphi_{A_{k}}^{k, f}$ from $A_{k}$ to $C_{k}$. Recall from (4.4) that it is sufficient to compute $A_{k}$ characters on $d \in \mathrm{C}_{k}$ or $d=a \mathrm{e}_{k}$ with $a \in \mathrm{A}_{k^{\prime}}$.

Proposition 4.3 For each $d \in \mathcal{A}_{k}$ and $0 \leq f \leq r \leq k$, we have

$$
\varphi_{\mathrm{A}_{k}}^{r, f}(d)= \begin{cases}\varphi_{\mathrm{C}_{k}}^{f}(d), & \text { if } r=k \text { and } \operatorname{rank}(d)=k, \\ 0, & \text { if } r=k \text { and } \operatorname{rank}(d)<k, \\ \varphi_{\mathrm{A}_{k^{\prime}}}^{r, f}(a), & \text { if } r<k \text { and } d=a \mathrm{e}_{k} \text { with } a \in \mathrm{A}_{k^{\prime}} .\end{cases}
$$

Proof If $r=k$ and $\operatorname{rank}(d)<k$, then by (4.18) $d$ acts as 0 on every $t \in \mathrm{I}_{\mathrm{A}_{k}}^{k, f}$ and thus $\varphi_{\mathrm{A}_{k}}^{k, f}(d)=0$. If $r=k$ and $\operatorname{rank}(d)=k$, then $d \in \mathrm{C}_{k}$. The restriction to diagrams of rank $r=k$ is exactly the action of $\mathrm{C}_{k}$ on $\mathrm{I}_{\mathrm{A}_{k}}^{k, f}=\mathrm{I}_{\mathrm{C}_{k}}^{f}$. When $\mathrm{C}_{k}=\mathbb{K} \mathrm{S}_{k}$ this is the Saxl representation as observed in Remark 4.4. In the planar case, $\mathrm{C}_{k}=\mathbb{K} \mathbf{1}_{k}$, the only planar rank $k$ diagram is $\mathbf{1}_{k}$ and we must have $k=f$.

Let $r<k$ and $d=a \mathrm{e}_{k}=\mathrm{e}_{k} a$. Then $t \in \mathrm{I}_{\mathrm{A}_{k}}^{r, f}$ contributes to the trace of $d$ only if $d \circ t \circ d^{T}=t$. Furthermore, $d \circ t \circ d^{T}=\left(\mathrm{e}_{k} a\right) \circ t \circ\left(\mathrm{e}_{k} a\right)^{T}=\mathrm{e}_{k} a \circ t \circ a^{T} \mathrm{e}_{k}^{T}=$ $\mathrm{e}_{k} a \circ t \circ a^{T} \mathrm{e}_{k}=a^{\prime} \mathrm{e}_{k}$ with $a^{\prime} \in \mathcal{A}_{k^{\prime}}$. Thus $t$ contributes to the trace only if $t=t^{\prime} \mathrm{e}_{k}$ for $t^{\prime} \in \mathrm{I}_{\mathrm{A}_{k}^{\prime}}^{r, f}$. Now, $d \cdot t=\left(a \mathrm{e}_{k}\right) \cdot\left(t^{\prime} \mathrm{e}_{k}\right)=x^{\kappa\left(a \mathrm{e}_{k}, t^{\prime} \mathrm{e}_{k}\right)} S\left(a \mathrm{e}_{k}, t^{\prime} \mathrm{e}_{k}\right)\left(a \mathrm{e}_{k}\right) \circ\left(t^{\prime} \mathrm{e}_{k}\right) \circ\left(a \mathrm{e}_{k}\right)^{T}=$ $x^{\kappa\left(a \mathrm{e}_{k}, t^{\prime} \mathrm{e}_{k}\right)} S\left(a \mathrm{e}_{k}, t^{\prime} \mathrm{e}_{k}\right)\left(a \circ t^{\prime} \circ a^{T}\right)\left(\mathrm{e}_{k} \circ \mathrm{e}_{k} \circ \mathrm{e}_{k}\right)=x^{\kappa\left(a \mathrm{e}_{k}, t^{\prime} \mathrm{e}_{k}\right)} S\left(a \mathrm{e}_{k}, t^{\prime} \mathrm{e}_{k}\right)\left(a \circ t^{\prime} \circ\right.$ $\left.a^{T}\right) \mathrm{e}_{k}$. Using the fact that both $a$ and $t^{\prime}$ commute with $\mathrm{e}_{k}$, we see that $x^{\kappa\left(a \mathrm{e}_{k}, t^{\prime} \mathrm{e}_{k}\right)}=$ $x^{\kappa\left(a, t^{\prime}\right)}$ and $S\left(a \mathrm{e}_{k}, t^{\prime} \mathrm{e}_{k}\right)=S\left(a, t^{\prime}\right)$. Therefore, $t$ contributes to the trace if and only if 
$t=t^{\prime} \mathrm{e}_{k}$ and, in this case, the $t-t$ entry of the action of $d$ on $\mathrm{M}_{\mathrm{A}_{k}}^{r, f}$ equals the $t^{\prime}-t^{\prime}$ entry of the action of $a$ on $\mathrm{M}_{\mathrm{A}_{k^{\prime}}}^{r, f}$. Thus $\varphi_{\mathrm{A}_{k}}^{r, f}(d)=\varphi_{\mathrm{A}_{k^{\prime}}}^{r, f}(a)$.

When $A_{k}$ is nonplanar, we have $C_{r}=\mathbb{K} S_{r}$, and the Saxl model (Theorem 3.1) satisfies

$$
\mathbf{M}_{\mathbf{C}_{r}}=\bigoplus_{f=0}^{r} \mathbf{M}_{\mathrm{C}_{r}}^{f} \cong \bigoplus_{f=0}^{r} \bigoplus_{\lambda \in \Lambda_{\mathrm{C}_{r}}^{f}} \mathrm{C}_{r}^{\lambda} \cong \bigoplus_{\lambda \in \Lambda_{\mathrm{C}_{r}}} \mathrm{C}_{r}^{\lambda}, \quad \text { with } \Lambda_{\mathrm{C}_{r}}=\bigsqcup_{f=0}^{r} \Lambda_{\mathrm{C}_{r}}^{f}
$$

where $\Lambda_{\mathrm{C}_{r}}^{f}$ is the set of partitions of $r$ with $f$ odd parts. Here $\mathrm{M}_{\mathrm{C}_{r}}^{f}=0$ and $\Lambda_{\mathrm{C}_{r}}^{f}=\emptyset$ if $r-2 f$ is not even. When $\mathrm{A}_{k}$ is planar, we have $\mathrm{C}_{r}=\mathbb{K} \mathbf{1}_{r}$. In this case, the model is trivial and satisfies (4.20) with $\mathrm{M}_{\mathrm{C}_{r}}^{f}=0$, if $f \neq r$, and $\mathrm{M}_{\mathrm{C}_{r}}^{r}=\mathrm{M}_{\mathrm{C}_{r}}=\mathbb{K} \mathbf{1}$. We have $\Lambda_{\mathrm{C}_{r}}^{f}=\emptyset$, if $r \neq f$, and $\Lambda_{\mathrm{C}_{r}}^{r}=\Lambda_{\mathrm{C}_{r}}^{r}=\{(r)\}$.

By (4.3), the irreducible $A_{k}$ modules are indexed by

$$
\Lambda_{\mathrm{A}_{k}}=\bigsqcup_{r=0}^{k} \Lambda_{\mathrm{C}_{r}}=\bigsqcup_{r=0}^{k} \bigsqcup_{f=0}^{r} \Lambda_{\mathrm{C}_{r}}^{f}
$$

Applying Proposition 4.2 and Proposition 4.3 to Theorem 4.1 gives the following theorem.

Theorem 4.2 (Model Representation of $\mathrm{A}_{k}$ ) For $k \geq 0$, let $\mathrm{A}_{k}$ be any of the diagram algebras defined in Sect. 2, with $x$ chosen such that $\mathrm{A}_{k}$ is semisimple. Let $\left\{\mathrm{A}_{k}^{\lambda} \mid \lambda \in \Lambda_{\mathrm{A}_{k}}\right\}$ denote a complete set of irreducible $\mathrm{A}_{k}$-modules. Then for each $0 \leq f \leq r \leq k$, we have

(a) $\mathrm{M}_{\mathrm{A}_{k}}^{r, f} \cong \bigoplus_{\lambda \in \Lambda_{\mathrm{C}_{r}}^{f}} \mathrm{~A}_{k}^{\lambda} \quad$ and $\quad \mathrm{M}_{\mathrm{A}_{k}}^{r}=\bigoplus_{f=0}^{r} \mathrm{M}_{\mathrm{A}_{k}}^{r, f} \cong \bigoplus_{\lambda \in \Lambda_{\mathrm{C}_{r}}} \mathrm{~A}_{k}^{\lambda}$,

(c) $\mathrm{M}_{\mathrm{A}_{k}}=\bigoplus_{r=0}^{k} \bigoplus_{f=0}^{r} \mathrm{M}_{\mathrm{A}_{k}}^{r, f} \cong \bigoplus_{\lambda \in \Lambda_{\mathrm{A}_{k}}} \mathrm{~A}_{k}^{\lambda}$,

where $\mathrm{M}_{\mathrm{A}_{k}}^{r}=0$ and $\mathrm{M}_{\mathrm{A}_{k}}^{r, f}=0$ if there do not exist symmetric diagrams in $\mathrm{A}_{k}$ of rank $r$ or of rank $r$ with $f$ fixed points.

Corollary 4.1 If $\mathrm{A}_{k}$ is planar, then $\mathrm{M}_{\mathrm{A}_{k}}^{r} \cong \mathrm{A}_{k}^{(r)}$ is irreducible, and thus $\mathrm{M}_{\mathrm{A}_{k}}=$ $\bigoplus_{r=0}^{k} \mathrm{M}_{\mathrm{A}_{k}}^{r}$ is a decomposition into irreducible $\mathrm{A}_{k}$-modules.

\section{Gelfand models for diagram algebras}

In this section we illustrate the combinatorics of our model representation for each diagram algebra. We classify and count the symmetric diagrams of each type according to 
their rank and number of fixed blocks. These form a basis for the model representation defined in Theorem 4.2. As above, we need $\mathbb{K}$ to be chosen such that the diagram algebra is semisimple. For example, we may choose $\operatorname{char}(\mathbb{K})=0$. In some cases, for example Rui's criterion [33] on $\operatorname{char}(\mathbb{K})$ for the semisimplicity of the Brauer algebra, it is known for which positive characteristics the algebra is semisimple.

\subsection{The partition algebra $\mathrm{P}_{k}(x)$}

The partition algebra $\mathrm{P}_{k}(x)$ is spanned by the set partitions $\mathcal{P}_{k}$ defined in Sect. 2.1 and has dimension equal to the Bell number $B(2 k)$. For $x \in \mathbb{K}$ such that $x \notin\{0,1, \ldots, 2 k-1\}, \mathrm{P}_{k}(x)$ is semisimple (see [25] or [17]) with irreducible modules indexed by partitions in the set

$$
\Lambda_{\mathrm{P}_{k}}=\{\lambda \vdash r \mid 0 \leq r \leq k\} .
$$

For each $0 \leq \ell \leq\lfloor r / 2\rfloor$ there exist symmetric partition algebra diagrams in $\mathrm{I}_{\mathrm{P}_{k}}^{r, f}$ of rank $r$ with $\ell$ blocks that are transposed (i.e., propagating, nonidentity blocks) and $f=r-2 \ell$ fixed blocks. The number of these symmetric diagrams is

$$
\operatorname{dim} \mathrm{M}_{\mathrm{P}_{k}}^{r, r-2 \ell}=\left|\mathrm{I}_{\mathrm{P}_{k}}^{r, r-2 \ell}\right|=\sum_{b=r}^{k} \mathbf{S}(k, b)\left(\begin{array}{l}
b \\
r
\end{array}\right)\left(\begin{array}{c}
r \\
2 \ell
\end{array}\right)(2 \ell-1) ! !
$$

where $\mathbf{S}(k, b)$ is a Stirling number of the second kind. This sum is justified as follows: first partition the top and bottom rows of a symmetric diagram identically into $b$ blocks in $\mathbf{S}(k, b)$ ways. Then choose $r$ of these blocks to be propagating, and from those $r$ blocks, choose $2 \ell$ of them to correspond to transpositions and match them up in $(2 \ell-1)$ !! ways. The remaining $r-2 \ell$ blocks are fixed.

The model representation for $\mathrm{P}_{k}(x)$ satisfies,

$$
\mathrm{M}_{\mathrm{P}_{k}}^{r, f}=\bigoplus_{\substack{\lambda \vdash k \\ \operatorname{odd}(\lambda)=f}} \mathrm{P}_{k}^{\lambda} \quad \text { and } \quad \mathrm{M}_{\mathrm{P}_{k}}=\bigoplus_{r=0}^{k} \bigoplus_{\ell=0}^{\lfloor r / 2\rfloor} \mathrm{M}_{\mathrm{P}_{k}}^{r, r-2 \ell}=\bigoplus_{\lambda \in \Lambda_{\mathrm{P}_{k}}} \mathrm{P}_{k}^{\lambda}
$$

If we let $\mathrm{p}_{k}=\left|\mathrm{I}_{\mathrm{P}_{k}}\right|=\sum_{r=0}^{k} \sum_{\ell=0}^{\lfloor r / 2\rfloor}\left|\mathrm{I}_{\mathrm{P}_{k}}^{r, r-2 \ell}\right|=\operatorname{dim} \mathrm{M}_{\mathrm{P}_{k}}$ denote the total number of symmetric diagrams in $\mathrm{P}_{k}(x)$, then $\mathrm{p}_{k}$ is the sum of the degrees of the irreducible $\mathrm{P}_{k}(x)$-modules (which can be found in [14,17,24]). The first few values of $\mathrm{p}_{k}$ are 1,2 , $7,31,164,999,6841,51790,428131$. The sequence $\mathrm{p}_{k}$ is [37, A002872], which equals the number of type- $B$ set partitions (see Remark 4.1), and has exponential generating function $e^{\left(e^{2 x}-3\right) / 2+e^{x}}=\sum_{k=0}^{\infty} \mathrm{p}_{k} \frac{x^{k}}{k !}$. This generating function is justified in [28] in formula $6\left(5^{\prime}\right)$ (with $p=2$ in the notation of [28]) and in [29] (with $\mathrm{p}_{k}=H_{2, k}$ in the notation of [29]). 


\subsection{The Brauer algebra $\mathrm{B}_{k}(x)$}

The Brauer algebra $\mathrm{B}_{k}(x)$ is spanned by the Brauer diagrams and has dimension $\operatorname{dim} \mathrm{B}_{k}(x)=(2 k-1)$ !!. For $x \in \mathbb{K}$ such that $x \notin\{x \in \mathbb{Z} \mid 4-2 k \leq x \leq k-2\}, \mathrm{B}_{k}(x)$ is semisimple (see [33]) with irreducible modules indexed by partitions in the set

$$
\Lambda_{\mathrm{B}_{k}}=\{\lambda \vdash(k-2 r) \mid 0 \leq r \leq\lfloor k / 2\rfloor\} .
$$

Symmetric Brauer diagrams consist of $\ell$ transpositions, $f$ fixed points, and $c$ contractions (symmetric pairs of horizontal edges) with and $f=k-2 c-2 \ell$. For example, the symmetric Brauer diagram,

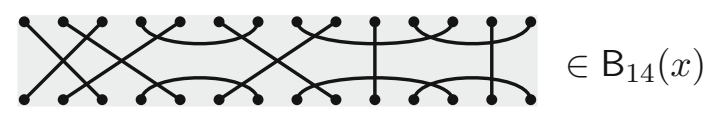

has $\ell=3$ transpositions $(1,3),(2,5),(6,9), c=3$ contractions in positions $\{4,7\},\{8,12\},\{11,14\}, f=2$ fixed points in positions 10 and 13 , and rank $r=8$. Symmetric Brauer diagrams have rank $r=k-2 c$, for $0 \leq c \leq\lfloor k / 2\rfloor$, and the number of symmetric diagrams of rank $r$ with with $f=r-2 \ell$ fixed points equals

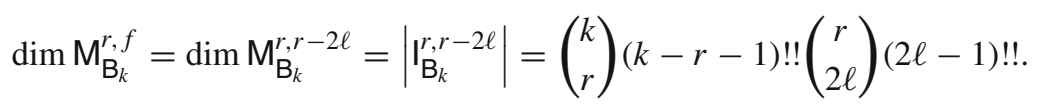

This count is justified as follows: choose the $r$ positions for the propagating edges in $\left(\begin{array}{l}k \\ r\end{array}\right)$ ways and pair the remaining $k-r$ positions for contractions in $(k-r-1)$ !! ways. Among the propagating edges, choose $r-2 \ell$ fixed points and pair the remaining edges in transpositions in $(2 \ell-1)$ !! ways.

The model representation for $\mathrm{B}_{k}(x)$ satisfies,

$$
\mathrm{M}_{\mathrm{B}_{k}}^{r, f} \cong \bigoplus_{\substack{\lambda \vdash r \\ \operatorname{odd}(\lambda)=f}} \mathrm{~B}_{k}^{\lambda} \quad \text { and } \quad \mathrm{M}_{\mathrm{B}_{k}} \cong \bigoplus_{c=0}^{\lfloor k / 2\rfloor} \bigoplus_{\ell=0}^{\lfloor(k-2 c) / 2\rfloor} \mathrm{M}_{\mathrm{B}_{k}}^{k-2 c, k-2 c-2 \ell} \cong \bigoplus_{\lambda \in \Lambda_{\mathrm{B}_{k}}} \mathrm{~B}_{k}^{\lambda}
$$

If we let $\mathrm{b}_{k}=\left|\mathrm{I}_{\mathrm{B}_{k}}\right|=\sum_{c=0}^{\lfloor k / 2\rfloor} \sum_{\ell=0}^{\lfloor(k-2 c) / 2\rfloor}\left|\mathrm{I}_{\mathrm{B}_{k}}^{k-2 c, k-2 c-2 \ell}\right|=\operatorname{dim} \mathrm{M}_{\mathrm{B}_{k}}$ denote the total number of symmetric diagrams in $\mathrm{B}_{k}(x)$, then $\mathrm{b}_{k}$ is the sum of the degrees of the irreducible $\mathrm{B}_{k}(x)$-modules (see [30]). This value can be obtained by summing (5.5) over the given values of $c$ and $\ell$ or by summing over $m=c+\ell$ as follows,

$$
\operatorname{dim} \mathrm{M}_{\mathrm{B}_{k}}=\sum_{m=0}^{\lfloor k / 2\rfloor}\left(\begin{array}{c}
k \\
2 m
\end{array}\right)(2 m-1) ! ! 2^{m}=\sum_{m=0}^{\lfloor k / 2\rfloor}\left(\begin{array}{c}
k \\
2 m
\end{array}\right) \frac{(2 m) !}{m !}=\sum_{m=0}^{\lfloor k / 2\rfloor}\left(\begin{array}{c}
k \\
2 m
\end{array}\right)\left(\begin{array}{c}
2 m \\
m
\end{array}\right) m ! .
$$

Here we choose $2 m$ points to be the endpoints of the transpositions and contractions, we pair them up in $(2 m-1)$ !! ways, and then we decide in $2^{m}$ ways if each is to be a transposition or a contraction. The first few values of $b_{k}$ are $1,1,3,7,25$, 81, 331, 1303, 5937, which is [37], A047974 with exponential generating function 
$b(x)=e^{x^{2}+x}=\sum_{k=0}^{\infty} \mathrm{b}_{k} \frac{x^{k}}{k !}$. To justify this generating function, verify that $\mathrm{b}_{k}$ satisfies the recurrence $\mathrm{b}_{k+2}=\mathrm{b}_{k+1}+(2 k+1) \mathrm{b}_{k}$ and therefore, $b^{\prime \prime}(x)=(1+2 x) b^{\prime}(x)+2 b(x)$ which has solution $b(x)=e^{x^{2}+x}$.

\subsection{The rook monoid algebra $\mathrm{R}_{k}$}

The rook monoid algebra $R_{k}$ is the subalgebra spanned by rook monoid diagrams with parameter $x=1$. It has dimension $\operatorname{dim} \mathrm{R}_{k}=\sum_{\ell=0}^{k}\left(\begin{array}{l}k \\ \ell\end{array}\right)^{2} \ell$ ! and is semisimple (see $[15,23,35])$ with irreducible modules labeled by

$$
\Lambda_{\mathrm{R}_{k}}=\{\lambda \vdash r \mid 0 \leq r \leq k\} .
$$

Symmetric rook monoid diagrams in $\mathrm{R}_{k}$ consist of $f$ fixed points, $\ell$ transpositions, and $k-f-2 \ell$ vertical pairs of empty vertices. For example, the symmetric rook monoid diagram,

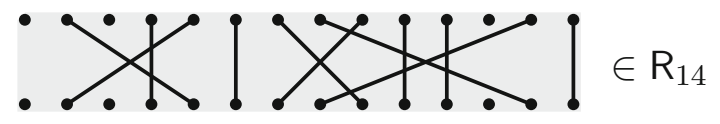

has $\ell=3$ transpositions $(2,5),(7,9),(8,13), f=5$ fixed points $4,6,10,11,14$, empty vertices in positions $1,3,12$, and rank $r=11$. Observe that $f=r-2 \ell$ and that every pair $0 \leq f \leq r \leq k$, with $r-f$ even, is possible. The number of symmetric rook diagrams of rank $r$ with $f=r-2 \ell$ fixed points is

$$
\operatorname{dim} \mathrm{M}_{\mathrm{R}_{k}}^{r, f}=\operatorname{dim} \mathrm{M}_{\mathrm{R}_{k}}^{r, r-2 \ell}=\left|\mathrm{I}_{\mathrm{R}_{k}}^{r, r-2 \ell}\right|=\left(\begin{array}{c}
k \\
r
\end{array}\right)\left(\begin{array}{c}
r \\
2 \ell
\end{array}\right)(2 \ell-1) ! !
$$

To justify this count, choose the $r$ positions for propagating edges in $\left(\begin{array}{l}k \\ r\end{array}\right)$ ways, choose $r-2 \ell$ positions for fixed points among these in $\left(\begin{array}{c}r \\ 2 \ell\end{array}\right)$ ways, and pair the remaining propagating edges into transpositions in $(2 \ell-1)$ !! ways.

The model representation for $\mathrm{R}_{k}$ satisfies,

$$
\mathrm{M}_{\mathrm{R}_{k}}^{r, f} \cong \bigoplus_{\substack{\lambda \vdash r \\ \operatorname{odd}(\lambda)=f}} \mathrm{R}_{k}^{\lambda} \quad \text { and } \quad \mathrm{M}_{\mathrm{R}_{k}} \cong \bigoplus_{r=0}^{k} \bigoplus_{\ell=0}^{\lfloor r / 2\rfloor} \mathrm{M}_{\mathrm{R}_{k}}^{r, r-2 \ell} \cong \bigoplus_{\lambda \in \Lambda_{\mathrm{R}_{k}}} \mathrm{R}_{k}^{\lambda}
$$

If we let $\mathrm{r}_{k}=\left|\mathrm{I}_{\mathrm{R}_{k}}\right|=\sum_{r=0}^{k} \sum_{\ell=0}^{\lfloor r / 2\rfloor}\left|\mathrm{I}_{\mathrm{R}_{k}}^{r, r-2 \ell}\right|=\operatorname{dim} \mathrm{M}_{\mathrm{R}_{k}}$ denote the total number of symmetric diagrams in $R_{k}$, then $r_{k}$ is the sum of the degrees of the irreducible $R_{k^{-}}$ modules (which can be found in $[15,35]$ ). The first few values of these dimensions are $1,2,5,14,43,142,499,1,850,7,193$. The sequence $r_{k}$ gives the number of "self-inverse partial permutations" and is [37, A005425]. Furthermore, $r_{k}$ is related to the number of involutions $s_{k}$ in the symmetric group (see Sect. 3.1) by the binomial transform $\mathrm{r}_{k}=\sum_{i=0}^{k}\left(\begin{array}{l}k \\ i\end{array}\right) \mathrm{s}_{i}$ and thus (see [12, (7.75)]) has exponential generating function $e^{x} e^{x^{2} / 2+x}=e^{x^{2} / 2+2 x}=\sum_{k=0}^{\infty} r_{k} \frac{x^{k}}{k !}$. 
Remark 5.1 The model representation that we construct with our methods here differs from the model for the rook monoid given in [23] in the same way that the Saxl symmetric group model differs from the one used by Adin et al. [1]. See Sect. 3.3.

\subsection{The rook-Brauer algebra $\mathrm{RB}_{k}(x)$}

The rook-Brauer algebra $\mathrm{RB}_{k}(x)$ is spanned by rook-Brauer diagrams and has dimension equal to $\sum_{\ell=0}^{k}\left(\begin{array}{c}2 k \\ 2 \ell\end{array}\right)(2 \ell-1)$ !! (see [6] or [26]). For all but finitely many $x \in \mathbb{K}$ (the exact values have not been determined), $\mathrm{RB}_{k}(x)$ is semisimple and its irreducible modules are labeled by

$$
\Lambda_{\mathrm{RB}_{k}}=\{\lambda \vdash r \mid 0 \leq r \leq\lfloor k\rfloor\} .
$$

Symmetric rook-Brauer diagrams in $\mathrm{RB}_{k}(x)$ consist of $\ell$ transpositions, $f$ fixed points, $c$ contractions, and $k-2 \ell-2 c-f$ vertical pairs of empty vertices. For example, the symmetric rook-Brauer diagram,

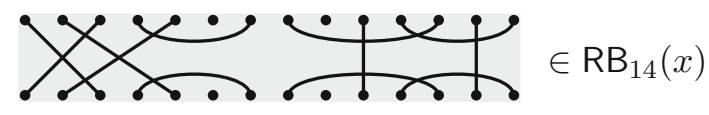

has $\ell=2$ transpositions $(1,3),(2,5), c=3$ contractions in positions $\{4,7\},\{8,12\}$, $\{11,14\}, f=2$ fixed points in positions 10 and 13 , empty vertices in positions 6 and 9 , and rank $r=6$. Observe that these diagrams satisfy $f=r-2 \ell$, and that every pair $0 \leq f \leq r \leq k$, with $r-f$ even, is possible. The number of symmetric diagrams of this type is

$$
\begin{aligned}
\operatorname{dim} \mathrm{M}_{\mathrm{RB}_{k}}^{r, f} & =\operatorname{dim} \mathrm{M}_{\mathrm{RB}_{k}}^{r, r-2 \ell}=\left|\begin{array}{l}
r, r-2 \ell \\
\mathrm{RB}_{k}
\end{array}\right| \\
& =\sum_{c=0}^{\lfloor(k-r) / 2\rfloor}\left(\begin{array}{l}
k \\
r
\end{array}\right)\left(\begin{array}{c}
k-r \\
2 c
\end{array}\right)(2 c-1) ! !\left(\begin{array}{c}
r \\
2 \ell
\end{array}\right)(2 \ell-1) ! !,
\end{aligned}
$$

where here we sum over the number $c$ of contractions. This count is justified as follows: in $\left(\begin{array}{l}k \\ r\end{array}\right)$ ways, choose $r$ positions for the propagating edges. Then from the non propagating points, select the $2 c$ endpoints for the contractions in $\left(\begin{array}{c}k-r \\ 2 c\end{array}\right)$ ways and match them up in $(2 c-1) !$ ! ways. Then choose the $2 \ell$ endpoints of the transpositions in $\left(\begin{array}{c}r \\ 2 \ell\end{array}\right)$ ways, and match them up in $(2 \ell-1)$ !! ways.

The model representation for $\mathrm{RB}_{k}(x)$ satisfies,

$$
\mathrm{M}_{\mathrm{RB}_{k}}^{r, f} \cong \bigoplus_{\substack{\lambda \vdash r \\ \operatorname{odd}(\lambda)=f}} \mathrm{RB}_{k}^{\lambda} \quad \text { and } \quad \mathrm{M}_{\mathrm{RB}_{k}} \cong \bigoplus_{r=0}^{k} \bigoplus_{\ell=0}^{\lfloor r / 2\rfloor} \mathrm{M}_{\mathrm{RB}_{k}}^{r, r-2 \ell} \cong \bigoplus_{\lambda \in \Lambda_{\mathrm{RB}_{k}}} \mathrm{RB}_{k}^{\lambda}
$$

If we let $\mathrm{rb}_{k}=\left|\mathrm{IRB}_{k}\right|=\sum_{r=0}^{k} \sum_{\ell=0}^{\lfloor r / 2\rfloor}||_{\mathrm{RB}_{k}}^{r, r-2 \ell} \mid=\operatorname{dim} \mathrm{M}_{\mathrm{RB}_{k}}$ denote the total number of symmetric diagrams in $\mathrm{RB}_{k}(x)$, then $\mathrm{rb}_{k}$ is the sum of the degrees of the irreducible $\mathrm{RB}_{k}(x)$-modules (these dimensions can be found in [6] or [26]). The first 
few values of $\mathrm{rb}_{k}$ are $1,2,6,20,76,312,1,384,6,512,32,400$. The sequence $\mathrm{rb}_{k}$ is [37, A000898] and it is related to the number of symmetric diagrams $b_{k}$ in the Brauer algebra by the binomial transform $\mathrm{rb}_{k}=\sum_{i=0}^{k}\left(\begin{array}{c}k \\ i\end{array}\right) \mathrm{b}_{i}$ and thus (see [12, (7.75)]) has exponential generating function $e^{x} e^{x^{2}+x}=e^{x^{2}+2 x}=\sum_{k=0}^{\infty} \mathrm{rb}_{k} \frac{x^{k}}{k !}$.

\subsection{The Temperley-Lieb algebra $\mathrm{TL}_{k}(x)$}

The Temperley-Lieb algebra $\mathrm{TL}_{k}(x)$ is spanned by planar Brauer diagrams and has dimension equal to the Catalan number $C_{k}=\frac{1}{k+1}\left(\begin{array}{c}2 k \\ k\end{array}\right)$. For $x \in \mathbb{K}$ that is not the root of $U_{k}(x / 2)$, where $U_{k}$ is the Chebyshev polynomial of the second kind, $\operatorname{TL}_{k}(x)$ is semisimple (see [40] or [20]) with irreducible modules indexed by

$$
\Lambda_{\mathrm{TL}_{k}}=\{k-2 \ell \mid 0 \leq \ell \leq\lfloor k / 2\rfloor\} .
$$

Symmetric Temperley-Lieb diagrams of rank $r$ have $f$ fixed points and $c$ contractions with $r=f=k-2 c$. For example,

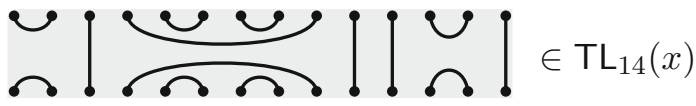

has $c=5$ contractions in positions $\{1,2\},\{4,9\},\{5,6\},\{7,8\},\{12,13\}, f=4$ fixed points in positions $3,10,11$, and 14 , and rank $r=4$. The number of symmetric Temperley-Lieb diagrams (see [40, p. 545] or [20, Sect. 5.1]) is given by

$$
\operatorname{dim} \mathrm{M}_{\mathrm{TL}_{k}}^{r, f}=\left|\mathrm{I}_{\mathrm{TL}_{k}}^{k-2 c}\right|=\left\{\begin{array}{l}
k \\
c
\end{array}\right\}:=\left(\begin{array}{l}
k \\
c
\end{array}\right)-\left(\begin{array}{c}
k \\
c-1
\end{array}\right) .
$$

The model representation for $\mathrm{TL}_{k}(x)$ satisfies,

$$
\mathrm{M}_{\mathrm{TL}_{k}}^{(k-2 c)} \cong \mathrm{TL}_{k}^{(k-2 c)} \quad \text { and } \quad \mathrm{M}_{\mathrm{TL}_{k}} \cong \bigoplus_{c=0}^{\lfloor k / 2\rfloor} \mathrm{M}_{\mathrm{TL}_{k}}^{(k-2 c)} \cong \bigoplus_{(k-2 c) \in \Lambda_{\mathrm{TL}_{k}}} \mathrm{TL}_{k}^{(k-2 c)}
$$

The total number of symmetric diagrams in $\mathrm{TL}_{k}(x)$ is

$$
\mathrm{tl}_{k}=\operatorname{dim} \mathrm{M}_{\mathrm{TL}_{k}}=\operatorname{dim}\left|\mathrm{I}_{\mathrm{TL}_{k}}\right|=\sum_{c=0}^{\lfloor k / 2\rfloor}\left|\mathrm{I}_{\mathrm{TL}_{k}}^{k-2 c}\right|=\sum_{c=0}^{\lfloor k / 2\rfloor}\left(\begin{array}{l}
k \\
c
\end{array}\right)-\left(\begin{array}{c}
k \\
c-1
\end{array}\right)=\left(\begin{array}{c}
k \\
\lfloor k / 2\rfloor
\end{array}\right),
$$

which are the central binomial coefficients [37, A000984], and the first few values are $1,1,2,3,6,10,20,35$, and 70 . This sequence has exponential generating function $I_{0}(2 x)+I_{1}(2 x)=\sum_{k=0}^{\infty} \mathrm{tl}_{k} \frac{x^{k}}{k !}$, where $I_{n}(z)$ is the modified Bessel function of the first kind (see for example [12, (5.78)]).

Remark 5.2 The irreducible modules $\mathrm{TL}_{k}^{(k-2 c)}$ are constructed in [40] on "cup diagrams" (or 1-factors). Cup diagrams correspond exactly to the upper half of a symmetric diagram (since the diagrams are symmetric, only half is needed), and the action of 
$\mathrm{TL}_{k}(x)$ on these diagrams is exactly the same as our conjugation action on symmetric diagrams.

\subsection{The Motzkin algebra $\mathrm{M}_{k}(x)$}

The Motzkin algebra $\mathrm{M}_{k}(x)$ is spanned by planar rook-Brauer diagrams, which correspond to partial planar matchings of $\left\{1, \ldots, k, 1^{\prime}, \ldots, k^{\prime}\right\}$, and so the dimension of $\mathrm{M}_{k}(x)$ is the Motzkin number $M_{2 k}$ (see [2]). For $x \in \mathbb{K}$ that is not the root of $U_{k}((x-1) / 2)$, where $U_{k}$ is the Chebyshev polynomial of the second kind, $\mathrm{M}_{k}(x)$ is semisimple (see [2]) and its irreducible modules are indexed by

$$
\Lambda_{\mathrm{M}_{k}}=\{0,1, \ldots, k\}
$$

Symmetric Motzkin diagrams of rank $r$ consist of $f=r$ fixed points, $c$ contractions, and $k-f-2 c$ pairs of empty vertices. For example, the symmetric Motzkin diagram,

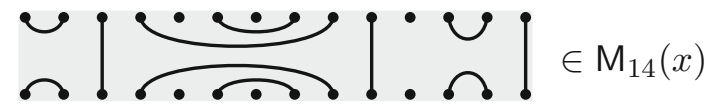

has $c=4$ contractions in positions $\{1,2\},\{4,9\},\{6,8\},\{12,13\}, f=3$ fixed points in positions $3,10,14$, vertical pairs of empty vertices in positions $5,7,11$, and rank $r=3$. Observe that every rank $0 \leq r \leq k$ is possible. The number of symmetric diagrams of this type is

$$
\operatorname{dim} \mathrm{M}_{\mathrm{M}_{k}}^{r}=\left|\mathrm{I}_{\mathrm{M}_{k}}^{r}\right|=\sum_{c=0}^{\lfloor(k-r) / 2\rfloor}\left(\begin{array}{c}
k \\
r+2 c
\end{array}\right)\left\{\begin{array}{c}
r+2 c \\
c
\end{array}\right\},
$$

where $\left\{\begin{array}{c}r+2 c \\ c\end{array}\right\}$ is defined in (5.15). This formula is derived in [2, (3.21)].

The model representation for $\mathrm{M}_{k}(x)$ satisfies,

$$
\mathrm{M}_{\mathrm{M}_{k}}^{r} \cong \mathrm{M}_{k}^{(r)} \quad \text { and } \quad \mathrm{M}_{\mathrm{M}_{k}} \cong \bigoplus_{r=0}^{k} \mathrm{M}_{\mathrm{M}_{k}}^{r} \cong \bigoplus_{r \in \Lambda_{\mathrm{M}_{k}}} \mathrm{M}_{k}^{(r)}
$$

If we let $\mathrm{m}_{k}=\left|\mathrm{I}_{\mathrm{M}_{k}}\right|=\sum_{r=0}^{k}||_{\mathrm{M}_{k}}^{r} \mid=\operatorname{dim} \mathrm{M}_{\mathrm{M}_{k}}$ denote the total number of symmetric diagrams in $\mathrm{M}_{k}(x)$, then $\mathrm{m}_{k}$ is the sum of the degrees of the irreducible $\mathrm{M}_{k}(x)$-modules. The first few values of $\mathrm{m}_{k}$ are 1, 2, 5, 13, 35, 96, 267, 750, 2123, 6046, and 17303 . The sequence $m_{k}$ is [37, A005773] and it is related to the number of symmetric diagrams $\mathrm{tl}_{k}$ in the Temperley-Lieb algebra by the binomial transform $\mathrm{m}_{k}=\sum_{i=0}^{k}\left(\begin{array}{l}k \\ i\end{array}\right) \mathrm{tl}_{i}$. Thus (see $[12,(7.75)]) \mathrm{m}_{k}$ has exponential generating function $e^{x}\left(I_{0}(2 x)+I_{1}(2 x)\right)=$ $\sum_{k=0}^{\infty} \mathrm{m}_{k} \frac{x^{k}}{k !}$.

Remark 5.3 The irreducible modules $\mathrm{M}_{k}^{(r)}$ are constructed in [2] on 1-factors. These 1 -factors correspond exactly to the upper half of a symmetric Motzkin diagram, and 
the action of $\mathrm{M}_{k}(x)$ on these diagrams is exactly the same as our conjugation action on symmetric diagrams. Indeed, it was knowledge of this conjugation action that allowed [2] to produce the action of $\mathrm{M}_{k}(x)$ on 1-factors.

\subsection{The planar rook monoid algebra $\mathrm{PR}_{k}$}

The planar rook monoid algebra $\mathrm{PR}_{k}$ is spanned by planar rook monoid diagrams with parameter set to $x=1$. It has dimension $\left(\begin{array}{c}2 k \\ k\end{array}\right)$, and is semisimple with irreducible modules labeled by

$$
\Lambda_{\mathrm{PR}_{k}}=\{0,1, \ldots, k\} .
$$

Symmetric planar rook monoid diagrams or rank $r$ consist of $f=r$ fixed points and $k-f$ vertical pairs of empty vertices. For example, the symmetric planar rook monoid diagram,

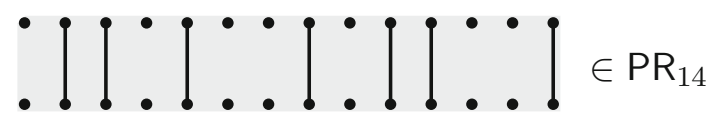

has $f=7$ fixed points in positions $2,3,5,8,10,11,14$, and rank $r=7$. We associate this diagram with its fixed points $S=\{2,3,5,8,10,11,14\}$, and thus symmetric diagrams correspond exactly to subsets $S \subseteq\{1,2, \ldots, k\}$. Thus, the number of symmetric diagrams is

$$
\operatorname{dim} \mathrm{M}_{\mathrm{PR}_{k}}^{r, f}=\operatorname{dim} \mathrm{M}_{\mathrm{PR}_{k}}^{r}=\left|\mathrm{I}_{\mathrm{PR}_{k}}^{r}\right|=\left(\begin{array}{l}
k \\
r
\end{array}\right) .
$$

The model representation for $\mathrm{PR}_{k}$ satisfies,

$$
\mathrm{M}_{\mathrm{PR}_{k}}^{r} \cong \mathrm{PR}_{k}^{(r)} \quad \text { and } \quad \mathrm{M}_{\mathrm{PR}_{k}} \cong \bigoplus_{r=0}^{k} \mathrm{M}_{\mathrm{PR}_{k}}^{r} \cong \bigoplus_{r \in \Lambda_{\mathrm{PR}_{k}}} \mathrm{PR}_{k}^{(r)}
$$

If we let $\mathrm{pr}_{k}=\left|\mathrm{IPR}_{k}\right|=\sum_{r=0}^{k}||_{\mathrm{PR}_{k}}^{r} \mid=\operatorname{dim} \mathrm{MPR}_{k}$ denote the total number of symmetric diagrams in $\mathrm{PR}_{k}$, then $\mathrm{pr}_{k}$ is the number of subsets of $\{1,2, \ldots, k\}$, so $\operatorname{pr}_{k}=\operatorname{dim} \mathrm{MPR}_{k}=2^{k}$ with exponential generating function $e^{2 x}=\sum_{k=0}^{\infty} 2^{k} \frac{x^{k}}{k !}$.

Remark 5.4 The irreducible modules $\mathrm{PR}_{k}^{(r)}$ are constructed in [9] on a basis of $r$ subsets of $\{1,2, \ldots, k\}$. These $r$-subsets correspond to symmetric rook monoid diagrams, and the action of $\mathrm{PR}_{k}$ on subsets is exactly the same as our conjugation action on symmetric diagrams. Indeed, it was knowledge of this conjugation action that led [9] to produce the action of $\mathrm{PR}_{k}$ on subsets.

Acknowledgments We thank Arun Ram for suggesting that we look for model representations of these algebras after seeing the dimension results in [16]. We also thank Michael Decker [5], whose honors project, under the direction T. Halverson, examined the model characters of the symmetric group and the partition algebra. It was during this collaboration that we constructed the combinatorial Saxl model for the symmetric group and conjectured the general construction of Gelfand models for diagram algebras. Upon the completion and submission of this manuscript, we learned of the preprint by V. Mazourchuk [27], who uses different methods to derive an analogous model to the one in this paper (see the comments in 
Sect. 3.3). We also thank the anonymous referees for several helpful recommendations. T. Halverson is supported in part by NSF Grant DMS-0800085. M. Reeks is supported in part by the Macalester College Anderson-Grossheusch Summer Research Fund.

\section{References}

1. Adin, R., Postnikov, A., Roichman, Y.: Combinatorial Gelfand models. J. Algebra 320, 1311-1325 (2008)

2. Benkart, G., Halverson, T.: Motzkin algebras. Eur. J. Comb. 36, 473-502 (2014)

3. Bernstein, I.N., Gelfand, I.N., Gelfand, I.M.: Models of representations of compact Lie groups (Russian). Funkc. Anal. i Priloz. 9(4), 61-62 (1975)

4. Brauer, R.: On algebras which are connected with the semisimple continuous groups. Ann. Math. 38, 85-872 (1937)

5. Decker, M.: A model representation of the symmetric group and the partition algebra, Macalester College Honors Thesis (2012)

6. del Mas, E., Halverson, T.: Representations of the Rook-Brauer algebra. Commun. Algebr. 42, 423-443 (2013)

7. Diaconis, P.: Group representations in probability and statistics. Institute of Mathematical Statistics Lecture Notes: Monograph Series, Vol. 11. Institute of Mathematical Statistics, Hayward (1988)

8. Diaconis, P., Holmes, S.: Random walks on trees and matchings. Electron. J. Probab. 7(6), 17 (2002)

9. Flath, D., Halverson, T., Herbig, K.: The planar rook algebra and Pascal's triangle. l'Enseign. Math. 54(2), 1-16 (2008)

10. Goodman, F.M., de la Harpe, P., Jones, V.F.R.: Coxeter Graphs and Towers of Algebras. Springer, New York (1989)

11. Goodman, F.M., Graber, J.: Celluarity and the Jones basic construction. Adv. Appl. Math. 46, 312-362 (2011)

12. Graham, R., Knuth, D., Patashnik, O.: Concrete Mathematics. A Foundation for Computer Science, 2nd edn. Addison-Wesley, Reading (1994)

13. Halverson, T., Ram, A.: Characters of algebras containing a Jones basic construction: the TemperleyLieb, Okada, Brauer, and Birman-Wenzl algebras. Adv. Math. 116, 263-321 (1995)

14. Halverson, T.: Characters of the partition algebra. J. Algebra 238, 502-533 (2001)

15. Halverson, T.: Representations of the $q$-rook monoid. J. Algebra 273, 227-251 (2004)

16. Halverson, T., Lewandowski, T.: RSK insertion for set partitions and diagram algebras. Electron. J. Comb. 11, $24(2004 / 06)$

17. Halverson, T., Ram, A.: Partition algebras. Eur. J. Comb. 26, 869-921 (2005)

18. Inglis, N., Richardson, R., Saxl, J.: An explicit model for the complex representations of $\mathrm{S}_{n}$. Arch. Math. 54, 258-259 (1990)

19. James, G., Kerber, A.: The Representation Theory of the Symmetric Group. Encyclopedia of Mathematics and its Applications, vol. 16. Addison-Wesley, Reading (1981)

20. Jones, V.F.R.: Index for subfactors. Invent. Math. 72, 1-25 (1983)

21. Jones, V.F.R.: The Potts model and the symmetric group, in: Subfactors. In: Proceedings of the Taniguchi Symposium on Operator Algebras (Kyuzeso, 1993), pp. 259-267. World Scientific Publishing, River Edge (1994)

22. Kljačko, A.A.: Models for complex representation of the groups $G L(n, q)$ and Weyl groups. Sov. Math. Dokl. 24, 469-499 (1981)

23. Kudryavtseva, G., Mazorchuk, V.: Combinatorial Gelfand models for some semigroups and $q$-rook monoid algebras. Proc. Edinb. Math. Soc. 52(2), 707-718 (2009)

24. Martin, P.P.: Temperley-Lieb algebras for non-planar statistical mechanics: the partition algebra construction. J. Knot Theory Ramif. 3, 51-82 (1994)

25. Martin, P.P., Saleur, H.: Algebras in higher-dimensional statistical mechanics-the exceptional partition (mean field) algebras. Lett. Math. Phys. 30, 179-185 (1994)

26. Martin, P.P., Mazorchuk, V.: On the representation theory of partial Brauer algebras. http://arxiv.org/ abs/1205.0464v1 (2012).

27. Mazorchuk, V.: Combinatorial Gelfand models for semisimple diagram algebras. Milan J. Math. 81, 385-396 (2013) 
28. Motzkin, T.: Sorting numbers for cylinders and other classification numbers. Combinatorics (Proceedings of Symposia in Pure Mathematics, Vol. 19, University of California, Los Angeles, 1968), pp. 167-176. American Mathematical Society, Providence (1971)

29. Quaintance, J.: Letter representations of $m \times n \times p$ proper arrays. Australas. J. Comb. 38, 289308 (2007)

30. Ram, A.: Characters of Brauer's centralizer algebras. Pac. J. Math 169, 173-200 (1995)

31. Reiner, V.: Non-crossing partitions for classical reflection groups. Discret. Math. 177, 195-222 (1997)

32. Reiner, V., Saliola, F., Welker, V.: Spectra of Symmetrized Shuffling Operators. Mem. Am. Math. Soc. 22 (2014)

33. Rui, H.: A criterion on the semisimple Brauer algebras. J. Comb. Theory Ser. A 111, 78-88 (2005)

34. Saxl, J.: On multiplicity-free permutation representations. In: Finite Geometries and Designs, London Mathematical Society Lecture Notes 49, 337-353 (1981)

35. Solomon, L.: Representations of the rook monoid. J. Algebra 256, 309-342 (2002)

36. Temperley, H.N.V., Lieb, E.H.: Relations between the "percolation" and the "colouring" problem and other graph-theoretical problems associated with regular planar lattices: some exact results for the "percolation" problem. Proc. R. Soc. Lond. Ser. A 322, 251-280 (1971)

37. The On-Line Encyclopedia of Integer Sequences. Published electronically at http://oeis.org

38. Thrall, R.M.: On symmetrized Kroneckeer powers and the structure of the free Lie ring. Am. J. Math. 64, 371-388 (1942)

39. Wenzl, H.: On the structure of Brauer's centralizer algebras. Ann. of Math. 128, 173-183 (1988)

40. Westbury, B.W.: The representation theory of the Temperley-Lieb algebras. Math. Z. 219, 539-565 (1995) 